\title{
Transverse momentum resummation for Higgs production via gluon fusion in the MSSM
}

\section{Robert V. Harlander, ${ }^{a}$ Hendrik Mantler ${ }^{b}$ and Marius Wiesemann ${ }^{c}$}

${ }^{a}$ Fachbereich C, Bergische Universität Wuppertal, 42097 Wuppertal, Germany

${ }^{b}$ TH Division, Physics Department, CERN, CH-1211 Geneva 23, Switzerland

${ }^{c}$ Physik-Institut, Universität Zürich, 8057 Zürich, Switzerland

E-mail: robert.harlander@uni-wuppertal.de, hmantler@cern.de, mariusw@physik.uzh.ch

ABSTRACT: The resummed transverse momentum distribution of supersymmetric Higgs bosons produced through gluon fusion at NLO + NLL is presented, including the exact quark and squark mass dependences. Considering various MSSM scenarios, we compare our results to previous ones within the POWHEG approach. We analyze the impact of the bottom loop which becomes the dominant contribution to the gluon fusion cross section for a wide range of the parameter space for the pseudo-scalar and heavy Higgs.

KeYwords: Higgs Physics, Supersymmetric Standard Model, Resummation, QCD

ARXIV EPRINT: 1409.0531 


\section{Contents}

1 Introduction 1

2 Transverse momentum resummation 3

2.1 Resummation and matching 3

2.2 The resummed cross section 4

$\begin{array}{lll}2.3 & \text { Components to the matched-resummed cross section } & 6\end{array}$

$\begin{array}{lll}3 & \text { Choosing the resummation scale } & 7\end{array}$

4 Input parameters $\quad 13$

$\begin{array}{lll}\text { 4.1 MSSM parameter points } & 13\end{array}$

$\begin{array}{lll}4.2 & \text { Theoretical uncertainties } & 14\end{array}$

5 Results $\quad 15$

6 Conclusions 23

A $g^{(n)}$-functions at NLL $\quad 24$

\section{Introduction}

After the observation of a Higgs boson of mass $125 \mathrm{GeV}[1,2]$, the measurement of its properties has become one of the central targets of the LHC. From the theoretical side, precise predictions for the production and decay rates of such a particle in various models are crucial to pin down its nature. An enormous effort has already gone into precision calculations of the total cross section as well as kinematical distributions (see refs. [3-5] for an overview).

In the Standard Model (SM), the Higgs is predominantly produced via gluon fusion, where the Higgs-gluon coupling is mediated by a quark loop. Its cross section is about an order of magnitude higher than the sum of all other processes, which retain their importance through their additional final state particles and/or their specific kinematics. The gluon fusion process has been studied in great detail over the recent years, leading to a significant decrease of the related theoretical uncertainties. In particular, the use of an effective theory approach for the calculation of higher order corrections allows, loosely speaking, to determine the cross section one perturbative order higher than in the full theory. Within this approach, also known as the heavy-top limit, the top quark is assumed to be infinitely heavy. The total cross section in this approximation is known up to next-to-next-toleading order (NNLO) [6-8] and even parts of the next-to-NNLO are known [9-12]. Electroweak corrections and further effects beyond NNLO have been evaluated in refs. [13-21] for 
example. The uncertainty induced by the heavy-top limit has been shown to be below $1 \%$ for the total rate at NNLO [22-26].

While the effect of the four lightest quarks as mediators of the gluon-Higgs coupling is negligible $(\lesssim 1 \%)$ and therefore usually omitted, the bottom quark contributes at the $5-10 \%$ level to the total cross section at next-to-leading order (NLO) [27, 28]. Due to the smallness of the bottom-quark mass, one cannot apply the same approximation as for the top-quark contributions to evaluate radiative corrections for the bottom loop, but typically includes the full quark mass dependence in the calculation.

Kinematical distributions of the Higgs boson provide an important handle on the determination of Higgs properties (see, e.g., refs. [29-31]). One of the most important differential observables in this respect is the transverse momentum $\left(p_{T}\right)$ distribution of the Higgs. Once sufficient statistics have been collected at the LHC, the comparison of the experimental result for this spectrum to its theoretical prediction in various models should allow for further restrictions of the allowed parameter space of these models.

In the SM, the NNLO transverse momentum distribution of the Higgs produced via gluon fusion at $p_{T}>0$ has already been known for some time in the heavy-top limit [3234]. ${ }^{1}$ Sub-leading top-mass effects have been considered in refs. [35, 36]. Furthermore, also the fully-differential cross section has been determined up to NNLO [37-39]. However, it is well known that those perturbative calculations break down for small transverse momenta due to the occurrence of logarithmically enhanced terms in $p_{T}$. Only a resummation of these terms to all orders in $\alpha_{s}$ provides a proper theoretical prediction at small values of $p_{T}$.

Transverse momentum resummation at leading logarithmic (LL) and next-to-LL (NLL) accuracy for the gluon fusion process in the heavy-top approximation has already been performed a long time ago [40-42]. Ref. [43] introduced a matching procedure to consistently combine the resummed distribution and the fixed order cross section valid at large $p_{T}$. Its application to the $p_{T}$ spectrum of the Higgs at NNLO+NNLL was implemented using the effective theory approach into the publicly available program HqT [43-45]. ${ }^{2}$

Finite top- and bottom-mass effects on the resummed $p_{T}$ spectrum have been considered in the POWHEG [48, 49] approach [50] and by analytic resummation through NLO+NLL $[47,51,52]$. The small bottom-quark mass $m_{\mathrm{b}}$ introduces an additional uncertainty because terms $\ln \left(m_{\mathrm{b}} / p_{T}\right)$ appear at the amplitude level ${ }^{3}$, which are potentially large and could spoil the collinear and soft approximation already at $p_{T} \gtrsim m_{\mathrm{b}}$ [47]. The small bottom Yukawa coupling in the SM prevents this uncertainty from becoming too severe though.

Supersymmetric extensions are among the most popular theories beyond the SM. The minimal supersymmetric SM (MSSM) contains two Higgs doublets, which lead to five phys-

\footnotetext{
${ }^{1}$ Throughout this paper, we consistently associate the $2 \rightarrow 1$ process $g g \rightarrow H$ with the leading-order (LO) $p_{T}$ distribution, although it only contributes at $p_{T}=0$.

${ }^{2} \mathrm{~A}$ Monte Carlo approach, based on the same resummation formalism, to add resummation effects to the differential NNLO cross section with respect to the Higgs and its decay products was implemented into the program HRes $[46,47]$.

${ }^{3}$ However, in the limit $p_{T} \rightarrow 0$, these terms $\sim \ln \left(m_{\mathrm{b}} / p_{T}\right)$ vanish, and therefore collinear and soft factorization is preserved.
} 
ical Higgs bosons, three of which are neutral and two charged. The production of the neutral MSSM Higgs bosons is typically dominated by either of two processes, gluon fusion or bottom-quark annihilation. For the theoretical status of the latter process, we refer the reader to ref. [53], where the resummed $p_{T}$ distribution through NNLO+NNLL was obtained, and the references therein. ${ }^{4}$

In this paper, we focus on the gluon fusion process in the MSSM, but our calculation will be applicable also in a general 2-Higgs doublet $\operatorname{model}^{5}$ (2HDM). The total Higgs production cross section in gluon fusion has been calculated up to NLO within the MSSM [50, 58-68]. The currently most accurate total cross section in the MSSM can be obtained with the publicly available program SusHi [69].

Our goal is the analytically resummed $p_{T}$ spectrum of all three neutral MSSM Higgs bosons produced through gluon fusion at NLO+NLL. Since the bottom Yukawa coupling can be significantly enhanced with respect to the SM, the issue of a proper treatment of bottom-quark induced effects on the cross section becomes more important. We propose a pragmatic way to separately set the resummation scale of these terms and to derive an estimate of the residual uncertainty.

We compare our results to the ones of a similar earlier study [50], which calculated the transverse momentum spectrum within the POWHEG approach in combination with a parton shower.

The paper is organized as follows: in section 2, we briefly review the formalism for the resummation of contributions at small transverse momenta in the gluon fusion process and discuss the required theoretical quantities. Our procedure for choosing the resummation scale is described in section 3. Section 4 lists the input parameters and defines a set of MSSM parameter points which we use for our analysis. It also describes the way we determine the theoretical uncertainties. Numerical results are presented in section 5 , where we analyze the $p_{T}$ spectra for all three neutral MSSM Higgs bosons in specific scenarios and the impact of the relative contributions ordered by the respective Yukawa couplings that enter the cross section. Section 6 contains our conclusions.

\section{Transverse momentum resummation}

\subsection{Resummation and matching}

Consider the transverse momentum distribution of a color-neutral heavy particle of mass $M$ produced via a $2 \rightarrow 1$ process in QCD. For $p_{T} \gtrsim M$, a fixed-order expansion of the cross section in the strong coupling $\alpha_{s}$ can be applied. In the limit $p_{T} \rightarrow 0$, however, large logarithms $\ln \left(p_{T} / M\right)$ appear at fixed order, which spoil the validity of the perturbative expansion. A proper prediction of the distribution at $p_{T} \ll M$ can be obtained by resumming these logarithms to all orders in $\alpha_{s}$. Following ref. [43], we split the $p_{T}$-dependent cross section as

$$
\frac{\mathrm{d} \sigma}{\mathrm{d} p_{T}^{2}}=\frac{\mathrm{d} \sigma^{(\mathrm{res})}}{\mathrm{d} p_{T}^{2}}+\frac{\mathrm{d} \sigma^{(\mathrm{fin})}}{\mathrm{d} p_{T}^{2}},
$$

\footnotetext{
${ }^{4}$ The NNLO $p_{T}$ distribution for bottom-quark annihilation is already known for a while [54-57].

${ }^{5}$ Concerning the total cross section in $2 \mathrm{HDMs}$, see ref. [70].
} 
where the resummed logarithmic contributions in $p_{T}$ are contained in the first term on the r.h.s., while the second term remains finite as $p_{T} \rightarrow 0$. Working at finite orders and splitting up the second term on the r.h.s. of eq. (2.1), the cross section can be cast into the following form:

$$
\left[\frac{\mathrm{d} \sigma}{\mathrm{d} p_{T}^{2}}\right]_{\text {f.o.+l.a. }}=\left[\frac{\mathrm{d} \sigma^{(\text {res })}}{\mathrm{d} p_{T}^{2}}\right]_{\text {l.a. }}+\left[\frac{\mathrm{d} \sigma}{\mathrm{d} p_{T}^{2}}\right]_{\text {f.o. }}-\left[\frac{\mathrm{d} \sigma^{(\text {res })}}{\mathrm{d} p_{T}^{2}}\right]_{\text {f.o. }},
$$

where "f.o." (=fixed order) denotes the perturbative, and "l.a." the logarithmic accuracy (to be defined below) under consideration. The imposed matching condition

$$
\left[\left[\frac{\mathrm{d} \sigma^{(\mathrm{res})}}{\mathrm{d} p_{T}^{2}}\right]_{\text {l.a. }}\right]_{\text {f.o }}=\left[\frac{\mathrm{d} \sigma^{(\mathrm{res})}}{\mathrm{d} p_{T}^{2}}\right]_{\text {f.o. }}
$$

defines the logarithmic accuracy needed at a specific perturbative order, and vice versa. In eq. (2.2), all terms $\sim \delta\left(p_{T}\right)$ are contained in the first term of the r.h.s.; in practical calculations, one can therefore disregard such terms in the second and third term since they will cancel among each other.

The matching procedure as proposed in ref. [43] induces a unitarity constraint on the matched cross section which implies that the integral over $p_{T}^{2}$ reproduces the total cross section $\sigma_{\text {tot }}$ at fixed order:

$$
\int \mathrm{d} p_{T}^{2}\left[\frac{\mathrm{d} \sigma}{\mathrm{d} p_{T}^{2}}\right]_{\text {f.o.+l.a. }} \equiv\left[\sigma_{\text {tot }}\right]_{\text {f.o. }}
$$

In the next section, we will address the evaluation of $\mathrm{d} \sigma^{(\mathrm{res})} / \mathrm{d} p_{T}^{2}$.

\subsection{The resummed cross section}

The resummation of large logarithmic contributions is performed in the impact parameter or $b$ space, given by the Fourier transform w.r.t. the transverse momentum: ${ }^{6}[72,73]$

$$
\begin{aligned}
\frac{\mathrm{d} \sigma^{F,(\mathrm{res})}}{\mathrm{d} p_{T}^{2}}=\tau \int_{0}^{\infty} \mathrm{d} b \frac{b}{2} J_{0}\left(b p_{T}\right) \sum_{c \in\{g, q, \bar{q}\}} \hat{\sigma}_{c \bar{c}}^{F,(0)} H_{c}^{F}\left(\alpha_{s}\right) S_{c}(M, b) \\
\quad \times \sum_{i, j \in\{g, q, \bar{q}\}}\left[C_{c i}\left(\alpha_{s}\left(b_{0} / b\right)\right) \otimes C_{\bar{c} j}\left(\alpha_{s}\left(b_{0} / b\right)\right) \otimes f_{i}\left(b_{0} / b\right) \otimes f_{j}\left(b_{0} / b\right)\right](\tau),
\end{aligned}
$$

with $q \in\{u, d, s, c, b\}$, a numerical constant ${ }^{7} b_{0}=2 \exp \left(-\gamma_{E}\right)=1.12292 \ldots$, and the Bessel function of the first kind $J_{0}(x)$ with $J_{0}(0)=1$. Here and in what follows, the superscript $F$

\footnotetext{
${ }^{6}$ Throughout this paper, parameters that are not crucial for the discussion will be suppressed in function arguments. Note that we refrain from including the spin correlation functions $G$ introduced in ref. [71] here and in what follows, since they are not required at the order considered in this paper.

${ }^{7} \gamma_{E}=-\Gamma^{\prime}(1)$ is the Euler constant.
} 
is attached to process specific quantities in order to distinguish them from universal ones. The symbol $\otimes$ indicates the convolution in the following sense:

$$
\left[C_{c i}\left(\alpha_{s}\left(b_{0} / b\right)\right) \otimes f_{j}\left(b_{0} / b\right)\right]\left(z_{3}\right) \equiv \int_{0}^{1} \mathrm{~d} z_{1} \int_{0}^{1} \mathrm{~d} z_{2} \delta\left(z_{3}-z_{1} z_{2}\right) C_{c i}\left(\alpha_{s}\left(b_{0} / b\right), z_{1}\right) f_{j}\left(z_{2}, b_{0} / b\right),
$$

where $f_{j}(x, q)$ denotes the density of parton $j$ with momentum fraction $x$ of the proton, and evaluated at momentum transfer $q$.

The central element of the resummation formula is the so-called Sudakov form factor

$$
S_{c}(M, b)=\exp \left\{L g_{c}^{(1)}\left(\alpha_{s} L\right)+g_{c}^{(2)}\left(\alpha_{s} L\right)+\sum_{l=3}^{\infty}\left(\frac{\alpha_{s}}{\pi}\right)^{l-2} g_{c}^{(l)}\left(\alpha_{s} L\right)\right\},
$$

which resums logarithms of the form $L=\ln \left(b^{2} M^{2} / b_{0}^{2}\right)$, while $\alpha_{s} L$ is treated as being of order unity. The order of the expansion in the exponent then defines the logarithmic accuracy. At leading logarithmic level, only $g_{c}^{(1)}$ has to be taken into account, at NLL also $g_{c}^{(2)}$ and so forth. We give their functional expressions up to the required order in this paper (i.e. $g_{c}^{(1)}$ and $g_{c}^{(2)}$ ) in appendix A.

Clearly, there is a certain amount of freedom in the separation between the "hard" and the "soft" region which can be parametrized by the so-called resummation scale $Q$. Unless indicated otherwise, we have set $Q \equiv M$ throughout this section; the generalization of the formulas to $Q \neq M$ and consequently $L=\ln \left(Q^{2} b^{2} / b_{0}^{2}\right)$ can be found in ref. [43]. In fact, the choice of the resummation scale for the gluon fusion process will be one of the central issues of this paper and will be discussed in more detail in section 3 .

The Born factor $\hat{\sigma}_{c \bar{c}}^{F,(0)}$ in eq. (2.5) is given by the parton level cross section at LO. In general, the sum over $c$ accounts for all LO subprocesses that can produce the considered colorless particle. In the gluon fusion process though, only $c=g$ is relevant. An explicit analytical expression for $\hat{\sigma}_{g g}^{F,(0)}$ for this process can be found in eq. (21) of ref. [69], for example. ${ }^{8}$

The resummation coefficients in eq. (2.5) can be expanded perturbatively:

$$
C_{c i}\left(\alpha_{s}, z\right)=\delta_{c i} \delta(1-z)+\sum_{n=1}^{\infty}\left(\frac{\alpha_{s}}{\pi}\right)^{n} C_{c i}^{(n)}(z), \quad H_{c}^{F}\left(\alpha_{s}\right)=1+\sum_{n=1}^{\infty}\left(\frac{\alpha_{s}}{\pi}\right)^{n} H_{c}^{F,(n)} .
$$

NLL accuracy requires the knowledge of these coefficients to first order in $\alpha_{s}$. Evidently, $\delta(1-z)$ terms in $C_{c j}^{(n)}$ can be shifted to $H_{c}^{F,(n)}$ for $n \geq 1$, and vice versa. A particular choice of these terms in $C_{c j}^{(n)}$ (equivalently, a particular choice of $H_{c}^{F}$ for one process) defines what is called a resummation scheme [43]. Within a particular resummation scheme, the coefficients $C_{c j}$ can be considered universal, while $H_{c}^{F}$ is process dependent. The entire process dependence in eq. (2.5) within a given resummation scheme is thus contained in $H_{c}^{F}$ and $\sigma_{c \bar{c}}^{F,(0)}$. We give the resummation coefficients in the $g g \rightarrow \phi$ scheme $(\phi \in\{h, H, A\})$, which is defined by setting

$$
H_{g}^{F}\left(\alpha_{s}\right) \equiv 1
$$

for this process.

\footnotetext{
${ }^{8}$ In the notation of ref. [69], it is $\sigma_{c \bar{c}}^{F,(0)} \equiv \sigma_{0}^{\phi}$.
} 
The $C$-coefficients in the $g g \rightarrow \phi$ scheme are [74]

$$
C_{g q}^{(1)}(z)=C_{g \bar{q}}^{(1)}(z)=\frac{C_{F}}{2} z, \quad C_{g g}^{(1)}(z)=\delta(1-z)\left(\frac{C_{A}}{2} \zeta_{2}+\frac{\mathcal{A}_{g}^{\phi, \text { virt }}}{4}\right)
$$

where again $q \in\{u, d, s, c, b\}$ and $\zeta_{2} \equiv \zeta(2)=\pi^{2} / 6$, with Riemann's $\zeta$ function. $\mathcal{A}_{g}^{\phi \text {,virt }}$ denotes the finite part of the virtual corrections as defined in eq. (38) of ref. [74], i.e.

$$
\mathcal{A}_{g}^{\phi, \text { virt }}=\left.2 \cdot C^{\phi}\right|_{\mu_{\mathrm{R}}=\mu_{\mathrm{F}}}
$$

with $C^{\phi}$ from eq. (27) in ref. [69].

It can be shown that the unitarity constraint of eq. (2.4) can be imposed by replacing

$$
L \rightarrow \widetilde{L} \equiv \ln \left(\frac{Q^{2} b^{2}}{b_{0}^{2}}+1\right)
$$

in eq. (2.7). In addition, this replacement reduces unjustified resummation effects at high transverse momenta, since $\widetilde{L}$ vanishes in the limit $b \rightarrow 0$ (i.e. $p_{T} \rightarrow \infty$ ), while the large- $b$ limit $\left(\right.$ small $\left.p_{T}\right)$ is preserved.

With the replacement in eq. (2.12) the resummed cross section $\mathrm{d} \sigma^{(\mathrm{res})} / \mathrm{d} p_{T}^{2}$ becomes explicitely $Q$ dependent; however, this dependence formally cancels between $\left[\mathrm{d} \sigma^{\text {(res) }} / \mathrm{d} p_{T}^{2}\right]_{\mathrm{l} \text {.a. }}$. and $\left[\mathrm{d} \sigma^{(\mathrm{res})} / \mathrm{d} p_{T}^{2}\right]_{\text {f.o. }}$ in eq. (2.2). Any residual dependence of the final result is beyond the specific logarithmic order under consideration. The variation of the cross section with $Q$ will be used to estimate the uncertainty due to missing terms of higher logarithmic accuracy.

\subsection{Components to the matched-resummed cross section}

The goal of this paper is to determine $p_{T}$ spectra of neutral MSSM Higgs bosons produced via gluon fusion by matching the NLO result to the resummed NLL approximation.

The relevant NLO matrix elements are taken from ref. [69], which include the SM-like contributions as well as sbottom, stop and gluino effects (see figure 1 for some sample Feynman diagrams). The LO diagrams, e.g. figure 1(a)-(c), determine the Born factor $\hat{\sigma}_{g g}^{\phi,(0)}$. The NLO $p_{T}$ distribution $\left[\mathrm{d} \sigma / \mathrm{d} p_{T}^{2}\right]_{\mathrm{f} . \mathrm{o} .=\mathrm{NLO}}$ in eq. $(2.2)$ at $p_{T}>0,{ }^{9}$ is governed by the real emission diagrams like the ones shown in figure $1(\mathrm{~h})$ and (i) (and similar ones with quark loops replaced by squark loops). Finally, the virtual diagrams, e.g. figure 1 (d)$(\mathrm{g})$, enter $C_{g g}^{(1)}$, as can be seen from eq. (2.10). These contributions allow to calculate $\left[\mathrm{d} \sigma^{(\mathrm{res})} / \mathrm{d} p_{T}^{2}\right]_{\text {l.a. }=\mathrm{NLL}}$.

The expansion of $\mathrm{d} \sigma^{(\mathrm{res})} / \mathrm{d} p_{T}^{2}$ with respect to $\alpha_{s}$ determines the logarithmic terms at NLO in eq. (2.2). The explicit expression can be found in eq. (72) of ref. [43], with the corresponding coefficients in eq. (63) and (64) of that paper.

The resummed expression $\left[\mathrm{d} \sigma^{(\mathrm{res})} / \mathrm{d} p_{T}^{2}\right]_{\mathrm{NLL}}$ has been calculated with a modified version of the program HqT [43-45], which determines the NNLO+NNLL $p_{T}$ distribution for the gluon fusion process using the approximation of an infinitely heavy top quark. We modified it for our purposes and implemented the resummation coefficients of eqs. (2.10) to include the MSSM effects.

\footnotetext{
${ }^{9}$ Note that $\delta\left(p_{T}\right)$ terms can be disregarded, see section 2.2.
} 


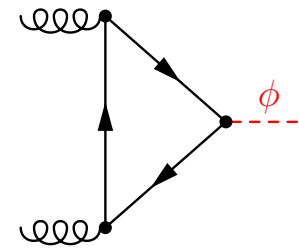

(a)

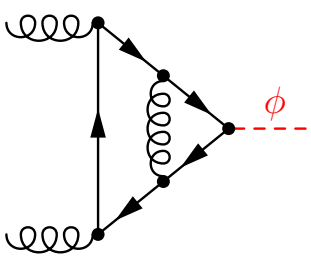

(d)

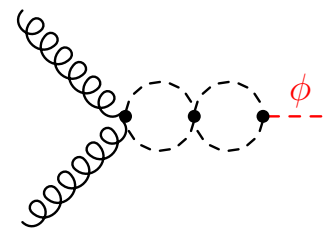

$(\mathrm{g})$

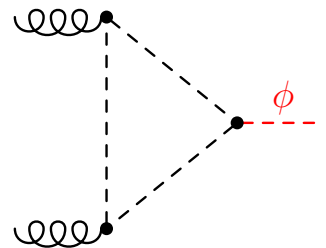

(b)

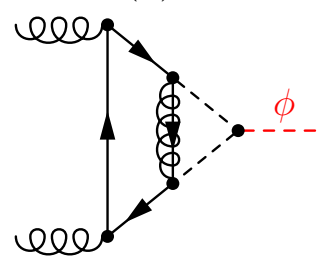

(e)

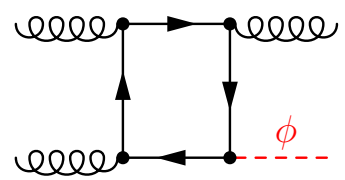

(h)

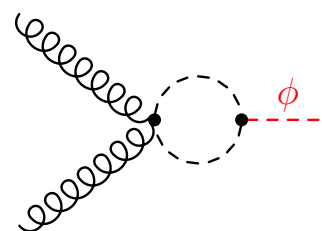

(c)

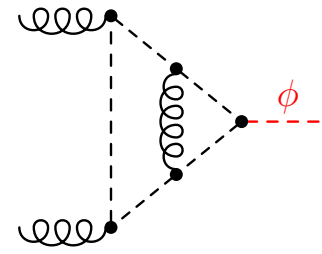

(f)

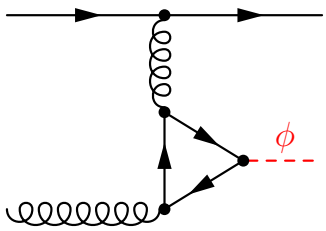

(i)

Figure 1. A sample of Feynman diagrams for $g g \rightarrow \phi$ contributing to the NLO cross section; (a-c) $\mathrm{LO},(\mathrm{d}-\mathrm{g})$ virtual and (h-i) real corrections. The graphical notation for the lines is: solid straight $\widehat{=}$ quark; spiraled $\widehat{=}$ gluon; dashed $\widehat{=}$ scalar (squark or Higgs); spiraled with line $\widehat{=}$ gluino.

\section{Choosing the resummation scale}

While the matched cross section is formally independent of the resummation scale $Q$, the actual numerical result can be quite sensitive to its particular choice due to the truncation at finite logarithmic order. It is therefore vital to determine an "optimal" choice for this scale. The resummation scale $Q$ can be viewed as a scale up to which resummation is extended. The soft and collinear approximation can be trusted only up to a finite value of $p_{T}$ which is determined by a characteristic external scale of the problem. Consequently, there is a maximum value of $p_{T}$ above which resummation is not valid and therefore, $Q$ should not be chosen beyond this value. Due to the constraint of eq. (2.4), a too large value of $Q$ not only spoils the prediction for $p_{T}<Q$, but also affects the large- $p_{T}$ region. ${ }^{10}$ The distribution can thus deviate significantly from the fixed-order prediction even in regions where the latter should provide a good approximation.

For the top-quark induced gluon-Higgs coupling, the characteristic scale is of the order of the Higgs mass $\left(m_{\phi}\right)$. Consequently, a reasonable range for phenomenological studies is $Q \in\left[m_{\phi} / 4, m_{\phi}\right]$, for example. The need for precise predictions requires one to take into account also bottom-quark induced effects to the gluon-Higgs coupling though [50, 51]. It has been shown [47] that terms $\sim \ln \left(m_{\mathrm{b}} / p_{T}\right)$ appear in the amplitude, which are potentially

\footnotetext{
${ }^{10} \mathrm{By}$ "large- $p_{T}$ ", we mean transverse momenta "of the order of the characteristic scale" and beyond.
} 
large and thus could spoil the soft and collinear approximation for $p_{T}>m_{\mathrm{b}}$. This has been used as an argument to choose $Q=m_{\mathrm{b}}$ [47], i.e. to effectively turn off Sudakov resummation at $p_{T} \gtrsim m_{\mathrm{b}}$, even though their actual impact has not been studied quantitatively in this context. However, these logarithms are not of Sudakov type as they vanish when $p_{T} \rightarrow 0$. In fact, they are closely related to logarithms $\ln \left(m_{\mathrm{b}} / m_{\phi}\right)$ which induce an uncertainty already at the level of the total cross section [27]. For a related quantity, namely the cross section with a veto on jets with $p_{T}^{\text {jet }}>p_{T \text {, veto }}^{\text {jet }}$, it was argued [52] that the impact of the analogous terms $\sim \ln \left(m_{\mathrm{b}} / p_{T, \text { veto }}^{\text {jet }}\right)$ remains moderate, and that one can treat these logarithms as a "finite remainder" together with all other finite terms (power corrections in $\left.p_{T, \text { veto }}^{\text {jet }}\right)$. A similar argument should apply also to the Higgs' $p_{T}$ spectrum.

In this section, we will formulate a pragmatic though quite general way to set the resummation scale. Rather than providing an ex-ante value of $Q$ for a particular process, our method is trial-and-error based and relies on simple expectations on the properties of the matched $p_{T}$ distribution. Roughly speaking, we determine the value of $Q$ as large as possible while requiring that the large- $p_{T}$ behavior of the matched distribution stays reasonably close to the fixed-order prediction.

More precisely, for Higgs masses up to $m_{\phi}=300 \mathrm{GeV}$, we determine $Q^{\max }$ as the maximum value of $Q$ for which the resummed $p_{T}$-distribution stays within the interval $[0,2] \cdot\left[\mathrm{d} \sigma / \mathrm{d} p_{T}^{2}\right]_{\text {f.o. }}$ for $p_{T} \in\left[m_{\phi}, p_{T}^{\max }\right]$. The restriction to the latter interval is needed because, on the one hand, resummation effects are expected to be large for smaller values of $p_{T}$; on the other hand, the numerical accuracy of our implementation of the resummation formula becomes unreliable above certain values of $p_{T}$. The specific value of $p_{T}^{\max }$ needs to be chosen case by case. For $m_{\phi}=125.6 \mathrm{GeV}$, it is $p_{T}^{\max } \approx 400 \mathrm{GeV}$, for $m_{\phi}=300 \mathrm{GeV}$, we use $p_{T}^{\max } \approx 650 \mathrm{GeV}$.

Neglecting squark effects for the moment, we apply this approach independently to the purely top and bottom induced contributions to the cross section, as well as to the top-bottom interference term. Figure 2 shows these three contributions to the resummed $p_{T}$-distribution for different resummation scales in the case of light Higgs production $\left(m_{h}=\right.$ $125.6 \mathrm{GeV}$ ), normalized to the respective fixed-order distribution. The curves for a heavy Higgs of $m_{H}=300 \mathrm{GeV}$ are shown in figure 3; those for a pseudo-scalar Higgs of the same mass are very similar to the latter, so we refrain from showing them here.

Larger Higgs masses correspond to a harder $p_{T}$ spectrum since the larger scale of the process leads to less soft gluon radiation. Nevertheless, for $m_{\phi}=800 \mathrm{GeV}$, the numerical accuracy of $\mathrm{d} \sigma^{(\mathrm{res})} / \mathrm{d} p_{T}^{2}$ becomes unreliable already at $p_{T} \gtrsim 700 \mathrm{GeV}$, so the above procedure for choosing $Q$ cannot be applied. We are therefore forced to modify our criterion for $m_{\phi}=800 \mathrm{GeV}$; our choice is to require $\left|\left[\mathrm{d} \sigma^{(\mathrm{res})} / \mathrm{d} p_{T}^{2}\right] /\left[\mathrm{d} \sigma / \mathrm{d} p_{T}^{2}\right]_{\text {f.o. }}-1\right|=1 / 2$ at $p_{T}=700 \mathrm{GeV}$. The corresponding curves for a heavy Higgs of $m_{H}=800 \mathrm{GeV}$ are shown in figure 4; again, those for a pseudo-scalar Higgs of the same mass are very similar, so we refrain from showing them here.

Our central scale choice is then defined as $Q_{0}=Q^{\max } / 2$, while the associated uncertainty is determined by varying $Q$ within the interval $\left[Q_{0} / 2,2 Q_{0}\right]$ (with an additional damping factor for large $p_{T}$, see section 4.2). The results of this procedure for a hadronic 


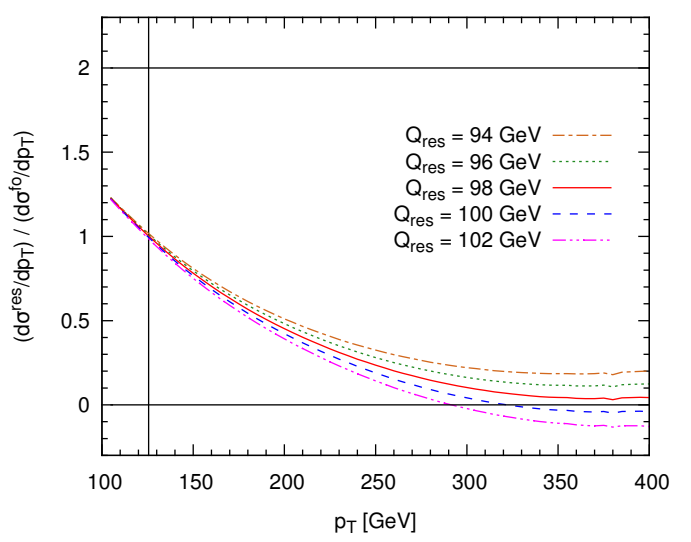

(a)

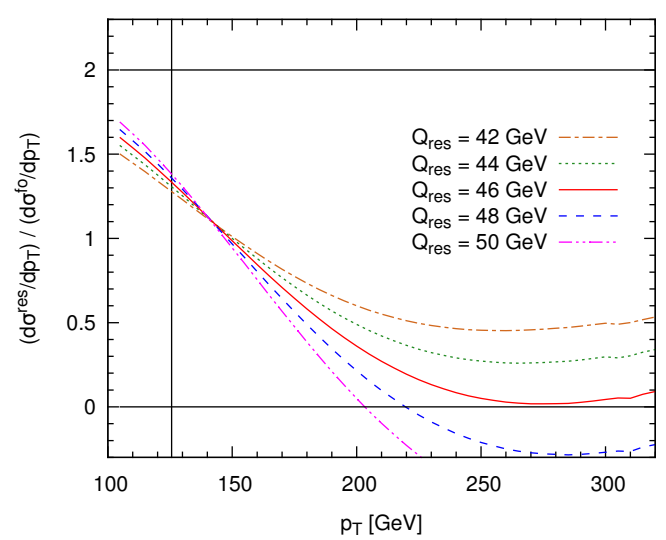

(b)

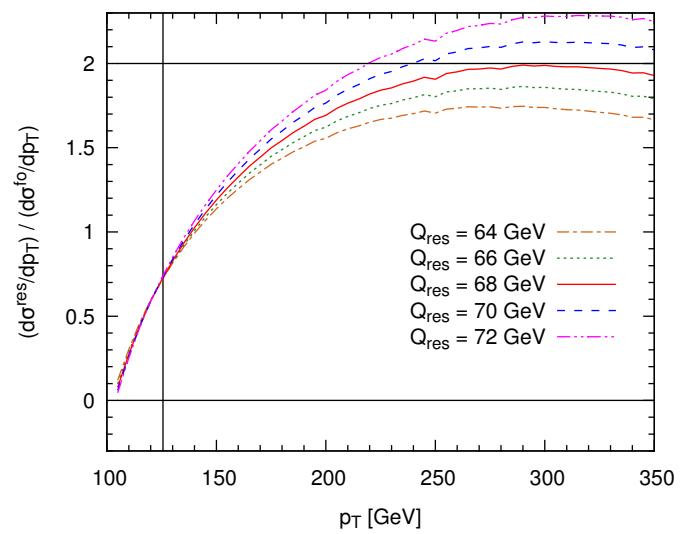

(c)

Figure 2. Large $p_{T}$-behavior of the cross section for a CP-even Higgs boson with $m_{h}=125.6 \mathrm{GeV}$. The different lines correspond to various choices of the resummation scale. (a) Pure top quark, (b) pure bottom quark, and (c) top-bottom interference contribution. The vertical line marks the value of the Higgs mass.

center of mass energy of $\sqrt{s}=13 \mathrm{TeV}$ are listed in table 1 . Somewhat reassuringly, for $m_{h}=125.6 \mathrm{GeV}$ our value for $Q_{0, t}$ agrees rather well with the default choice $Q=m_{h} / 2$ of ref. [45]. On the other hand, our interval for $Q_{0, b}$ extends to significantly larger values as the one argued for in ref. [47]. This is even more so for the interference term for which, in our case, the central resummation scale is almost the exact average of the $Q_{0, t}$ and $Q_{0, b}$, while ref. [47] fully attributed this term to the bottom contribution.

Our result for $Q_{0, \text { int }}$ agrees very well with what was found for the case of jet-veto in ref. [52]. By analyzing the finite remainder of the bottom contribution, which includes the top-bottom interference in their case, they find $Q \approx 35 \mathrm{GeV}$ to be an appropriate scale choice. ${ }^{11}$

Even though our approach to determine $Q$ seems very pragmatic, the underlying idea is physical, of course. A too large resummation scale $Q$ would overemphasize the Sudakov contribution, typically overshooting the cross section for $p_{T} \lesssim Q$. Due to the constraint of eq. (2.4), the only way to compensate for this effect is to reduce the cross section at larger

\footnotetext{
${ }^{11}$ Note that in the SM the bottom contribution is clearly dominated by the top-bottom interference term.
} 


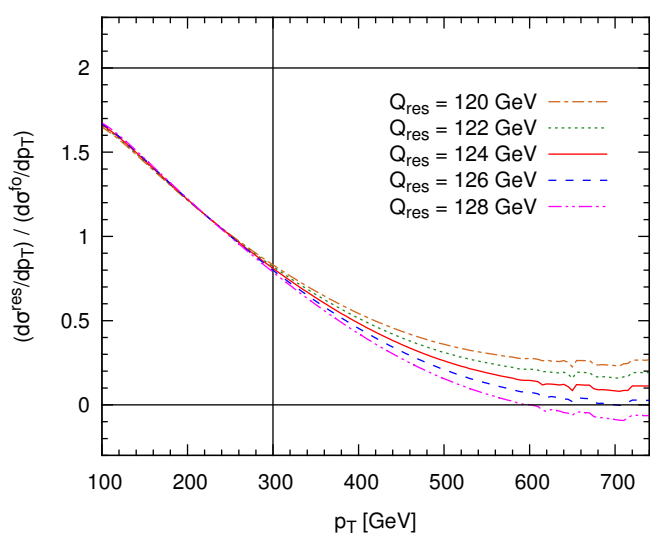

(a)

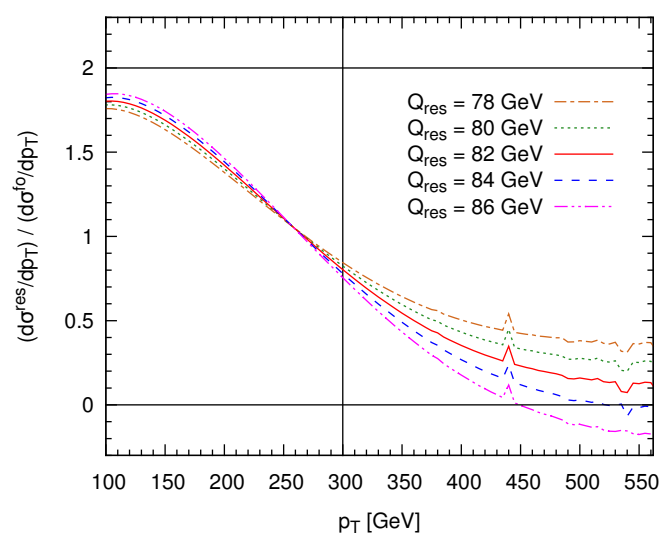

(b)

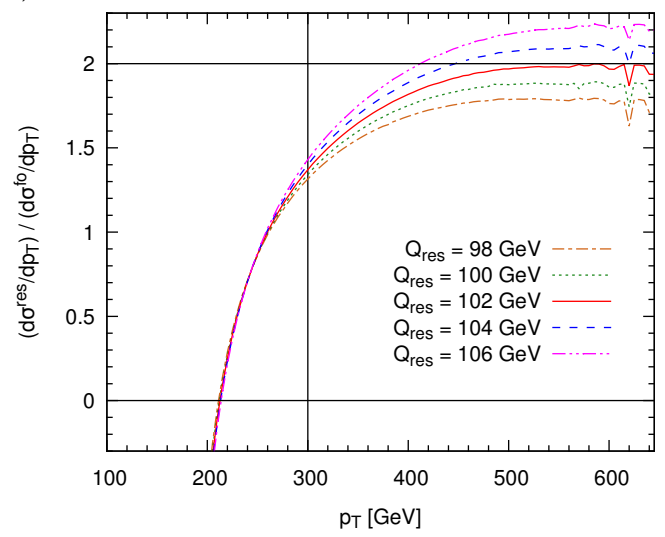

(c)

Figure 3. Same as figure 2, but for $m_{H}=300 \mathrm{GeV}$.

\begin{tabular}{|c|c|c|c|c|}
\hline Higgs type & mass $/ \mathrm{GeV}$ & $Q_{0, t} / \mathrm{GeV}$ & $Q_{0, b} / \mathrm{GeV}$ & $Q_{0, \text { int }} / \mathrm{GeV}$ \\
\hline \multirow{3}{*}{ scalar } & 125.6 & 49 & 23 & 34 \\
& 300 & 62 & 41 & 51 \\
& 800 & 107 & 77 & 105 \\
\hline \multirow{2}{*}{ pseudo-scalar } & 300 & 61 & 43 & 49 \\
& 800 & 117 & 80 & 104 \\
\hline
\end{tabular}

Table 1. Central resummation scales for the top-, bottom-, and their interference contribution to the cross section for scalar and pseudo-scalar Higgs production at various Higgs masses.

transverse momenta, such that it may even become negative. Therefore, by demanding resummation scales that lead to satisfactory matching at high transverse momenta, one indirectly restricts resummation to regions where the soft and collinear factorization is valid. More precisely, we can expect $Q$ to be close to the upper boundary of the range allowed by factorization, certainly not far above that. Note also that there is a certain amount of freedom how this range is defined. 


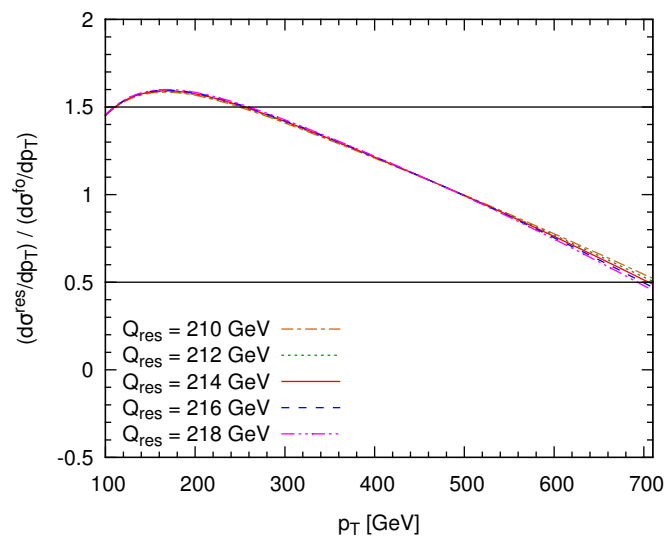

(a)

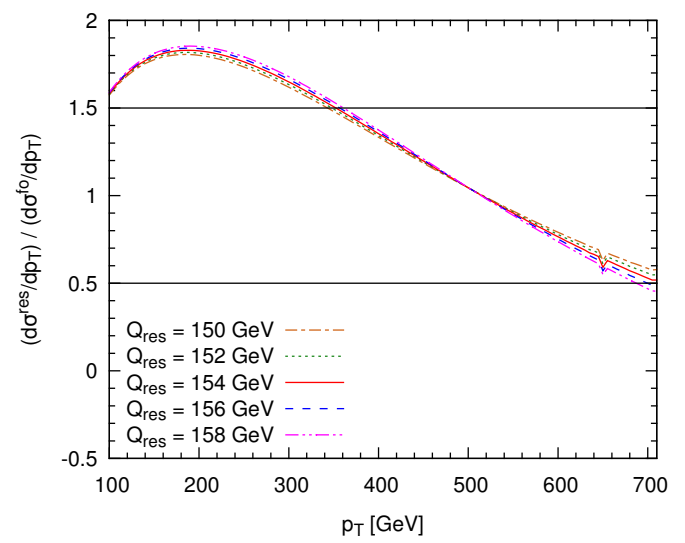

(b)

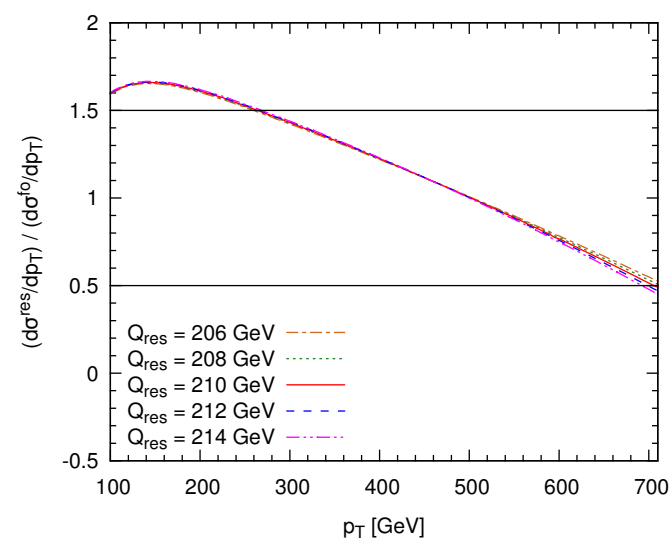

(c)

Figure 4. Same as figure 2, but for $m_{H}=800 \mathrm{GeV}$.

For our later discussion, it will be useful to study the impact of the top-, bottom- and their interference contribution on the shape of the $p_{T}$ distribution, i.e.

$$
\frac{1}{\sigma} \frac{\mathrm{d} \sigma}{\mathrm{d} p_{T}} \equiv \frac{\mathrm{d} \bar{\sigma}}{\mathrm{d} p_{T}}, \quad \text { with } \quad \int \mathrm{d} p_{T} \frac{\mathrm{d} \bar{\sigma}}{\mathrm{d} p_{T}}=1
$$

Figure 5 shows this shape of the bottom- and the top-bottom interference contribution relative to the top contribution, for scalar and pseudo-scalar Higgs production and three different values of the Higgs boson mass. In all cases, the bottom-quark distribution is significantly softer than the top contribution, but the difference between the two decreases for larger Higgs masses. This behavior is expected since soft radiation off the quark loop becomes larger for a smaller quark mass, or, equivalently, larger Higgs mass [75].

The shape of top-bottom interference term experiences a number of qualitative and quantitative changes as the Higgs mass increases. For $m_{\phi}=125.6 \mathrm{GeV}$, it can lead to quite some deviations from a pure top- or bottom-dominated shape, see figure 5 (a). Whether the spectrum becomes harder or softer depends on the sign of the interference (and thus also on the sign of the Yukawa couplings). ${ }^{12}$ Note also that there is a sign change at about

\footnotetext{
${ }^{12}$ Note that when subtracting a softer spectrum from a harder one, the combined spectrum is harder than both of them.
} 


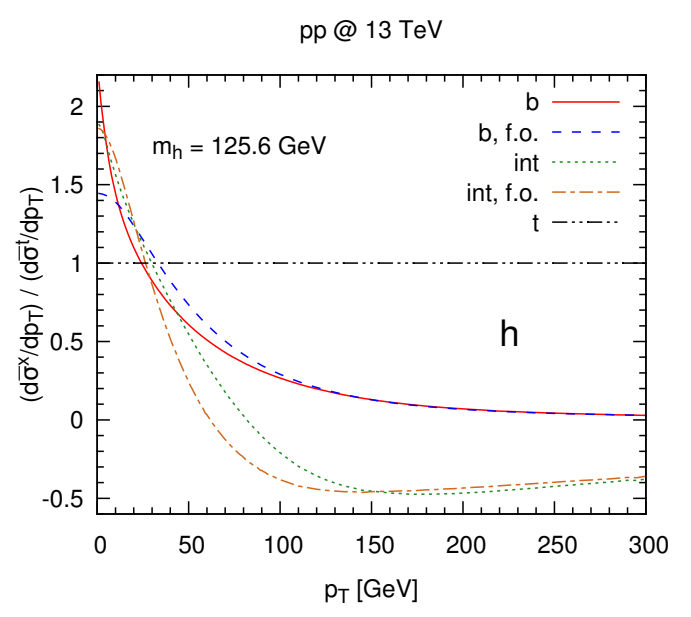

(a)

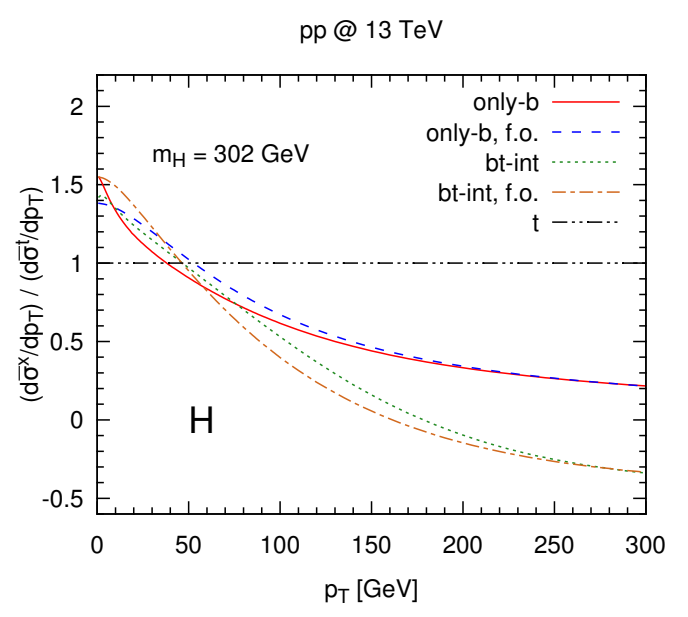

(b)

pp @ $13 \mathrm{TeV}$

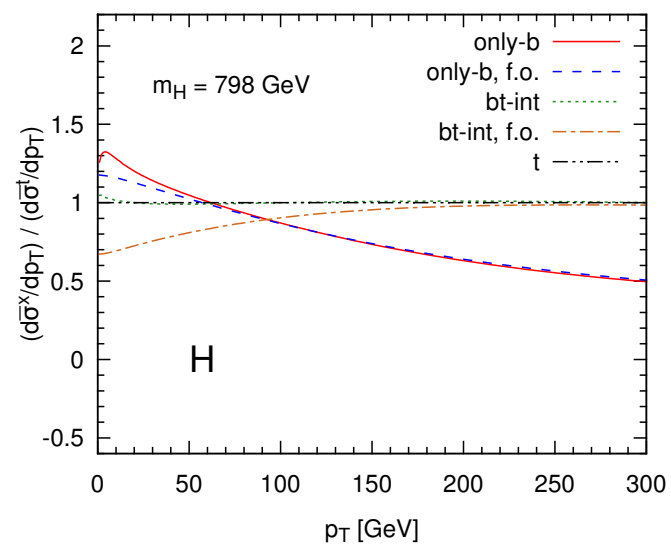

(c)

Figure 5. The $p_{T}$-shape for the bottom-quark (red, solid) and the top-bottom interference contribution (green, dotted), normalized to the top-contribution (black, dash-double dotted). Resummation scales are set as in table 1 . Also shown are the respective ratios for the NLO fixed-order results. (a-c) $m_{\phi}=125.6 / 300 / 800 \mathrm{GeV}$.

$p_{T}=80 \mathrm{GeV}$. At $m_{\phi}=300 \mathrm{GeV}$, figure $5(\mathrm{~b})$, the qualitative behavior remains roughly the same, but appears to be less distinct. At $m_{\phi}=800 \mathrm{GeV}$, figure 5 (c), on the other hand, the shape of the interference term is almost indistinguishable from the top contribution, except for very small $p_{T}$.

We observe a nice convergence of the resummed and the fixed-order distributions towards large $p_{T}$, as required by our determination of the matching scale. The curves for a pseudo-scalar Higgs are very similar to the scalar case which is why we refrain from showing them here.

As squark effects are typically small due to the fact that squark masses are expected to be of the order of a few hundred $\mathrm{GeV}$, we do not determine separate resummation scales for them. We therefore split the cross section into three terms: 
- The pure- $b$ contribution is proportional to the square of the bottom-Higgs coupling: $\sigma_{\text {pure- } b} \sim y_{b}^{2}$. Note that $\sigma_{\text {pure- } b}$ does not include sbottom effects.

- The int-b contribution is linearly proportional to the bottom Yukawa coupling: $\sigma_{\text {int- } b} \sim y_{b}$. It therefore contains interference terms of the bottom- with the topand squark-loop induced amplitude.

- The no-b contribution is defined as the cross section for $y_{b}=0$ and contains top- and squark-loop induced terms.

For the pure- $b$, the int- $b$, and the no- $b$ contribution, we use the resummation scales $Q_{0, b}$, $Q_{0, \text { int }}$, and $Q_{0, t}$ of table 1 , respectively, despite the fact that these scales were determined by disregarding squark effects. We have checked though that the numerical values of $Q_{0 \text {,int }}$ and $Q_{0, t}$ are hardly affected when squark effects are taken into account.

\section{Input parameters}

We present results for the resummed transverse momentum distribution of neutral Higgs bosons produced at the LHC via gluon fusion in various scenarios of the MSSM. Our goal here is not a detailed and comprehensive study of the $p_{T}$-spectrum in each of these scenarios though. Rather, we will make use of specific scenarios to highlight various features and dependences of the $p_{T}$-spectrum. Our default choice for the center-of-mass energy is $13 \mathrm{TeV}$. The central factorization and renormalization scale is set to $\mu_{\mathrm{F}}=\mu_{\mathrm{R}}=m_{\phi} / 2$. The choice for the central resummation scale is more subtle and is given in table 1. All numbers are obtained with the NLO PDF set of MSTW2008 [76], which implies that the input value for the strong coupling constant is taken as $\alpha_{s}\left(m_{Z}\right)=0.12018$. We use the on-shell top and bottom mass with numerical values $m_{\mathrm{t}}=173.2 \mathrm{GeV}$ and $m_{\mathrm{b}}=4.92 \mathrm{GeV}$ both for the internal propagators and the Yukawa couplings. Terms enhanced by $\tan \beta$ are implicitely resummed [77-81] by reweighing the bottom Yukawa coupling as described in ref. [82]. Similarly, the stop and the sbottom masses and mixing angles are renormalized as in ref. [82], in accordance with the definition of the benchmark scenarios to be described in the next section.

\subsection{MSSM parameter points}

We compare results for various MSSM benchmark scenarios, as defined in ref. [83 ${ }^{13}$ and refer to that paper for further details. These benchmark scenarios require the choice of $m_{A}$ and $\tan \beta$. Using the exclusion plots of ref. [83] and HiggsBounds [84-86], we identified proper (i.e. not yet excluded) parameter choices within the $m_{A}$-tan $\beta$ plane, while requiring that $m_{h}=125.6 \pm 0.7 \mathrm{GeV}$ (except for the light-stop scenario). The scenarios used for our analysis in section 5 are defined in table 2 .

\footnotetext{
${ }^{13}$ For the light-stop scenario we use the modified version suggested in ref. [82].
} 


\begin{tabular}{|c|c|c|c|c|}
\hline scenario & $m_{A} / \mathrm{GeV}$ & $\tan \beta$ & $m_{h} / \mathrm{GeV}$ & $m_{H} / \mathrm{GeV}$ \\
\hline \multirow{2}{*}{$\tau$-phobic } & 800 & 16 & 125.0 & 798.3 \\
\hline & 800 & 29.5 & 124.9 & 798.4 \\
\hline light-stau & 500 & 12 & 125.6 & 500.2 \\
\hline \multirow{3}{*}{$m_{h}^{\bmod +}$} & 500 & 17 & 125.6 & 499.9 \\
\hline & 800 & 17 & 125.6 & 800.0 \\
\hline & 800 & 40 & 126.1 & 799.7 \\
\hline \multirow{3}{*}{$m_{h}^{\bmod -}$} & 500 & 16.5 & 125.6 & 499.8 \\
\hline & 800 & 16.5 & 125.6 & 800.0 \\
\hline & 800 & 40 & 126.2 & 799.6 \\
\hline \multirow{2}{*}{$m_{h}^{\max }$} & 300 & 6.5 & 125.7 & 302.1 \\
\hline & 800 & 6.5 & 125.3 & 800.6 \\
\hline light-stop & 800 & 30 & 123.0 & 798.9 \\
\hline
\end{tabular}

Table 2. Parameter points considered in this paper. The full definition of the scenarios is given in ref. [83]; for the light-stop scenario, however, we modify the soft SUSY breaking wino and bino mass terms as well as the $\mu$-parameter as suggested in ref. [82], where $m_{2}=\mu=400 \mathrm{GeV}$ and $m_{1}=340 \mathrm{GeV}$, in order to evade constraints on the stop and sbottom masses presented by ATLAS and CMS [87-89]. The particular parameter points defined here will be refered to in the text as "scenario $\left(m_{A} / \mathrm{GeV}, \tan \beta\right)$ "; for example, the first parameter point in the table is $\tau$-phobic $(800,16)$ in this notation. The Higgs masses are evaluated with FeynHiggs [90-98] which we also apply to determine the corresponding Higgs couplings in the various scenarios.

\subsection{Theoretical uncertainties}

The main sources of theoretical uncertainty on our result for the $p_{T}$ distribution are due to missing higher order effects, as well as the uncertainty from the PDFs and $\alpha_{s}\left(m_{Z}\right)$. The latter two are usually estimated by following the so-called PDF4LHC recipe [99]. They will, however, not be part of our analysis within this manuscript.

The former are typically estimated by a variation of unphysical scales that emerge at finite perturbative or logarithmic order. In our case, these are the renormalization, the factorization, and the resummation scale. While for $\mu_{\mathrm{F}}$ and $\mu_{\mathrm{R}}$, we follow the standard procedure of considering the maximum variation of the cross section when $2 \mu_{\mathrm{R}} / m_{\phi}$ and $2 \mu_{\mathrm{F}} / m_{\phi}$ are taken from the set $\{1 / 2,1,2\}$, while excluding the values for which $\mu_{\mathrm{R}} / \mu_{\mathrm{F}} \in$ $\{1 / 4,4\}$. The impact of the choice of the resummation scale, on the other hand, we estimate by varying $Q / Q_{0}$ within the interval $[1 / 2,2]$. However, a variation within this region at large $p_{T}$ would grossly overestimate the uncertainty at $p_{T} \gtrsim m_{\phi}$, where the prediction should be well described by the fixed-order distribution. We therefore modulate the error band resulting from $Q$-variation by a damping factor

$$
d\left(p_{T}\right)=\left[1+\exp \left(\alpha\left(p_{T}-m_{\phi}\right)\right)\right]^{-1}, \quad \alpha=0.1 \mathrm{GeV}^{-1},
$$

which effectively switches off the $Q$-uncertainty for $p_{T} \gtrsim m_{\phi}$. Finally, we add the uncertainty estimated from $\mu_{\mathrm{F}^{-}}$and $\mu_{\mathrm{R}}$-variation and the one induced by $Q$ in quadrature. 
$\mathrm{SM}, \mathrm{m}_{\mathrm{H}}=125.6 \mathrm{GeV}, \mathrm{pp} @ 13 \mathrm{TeV}$

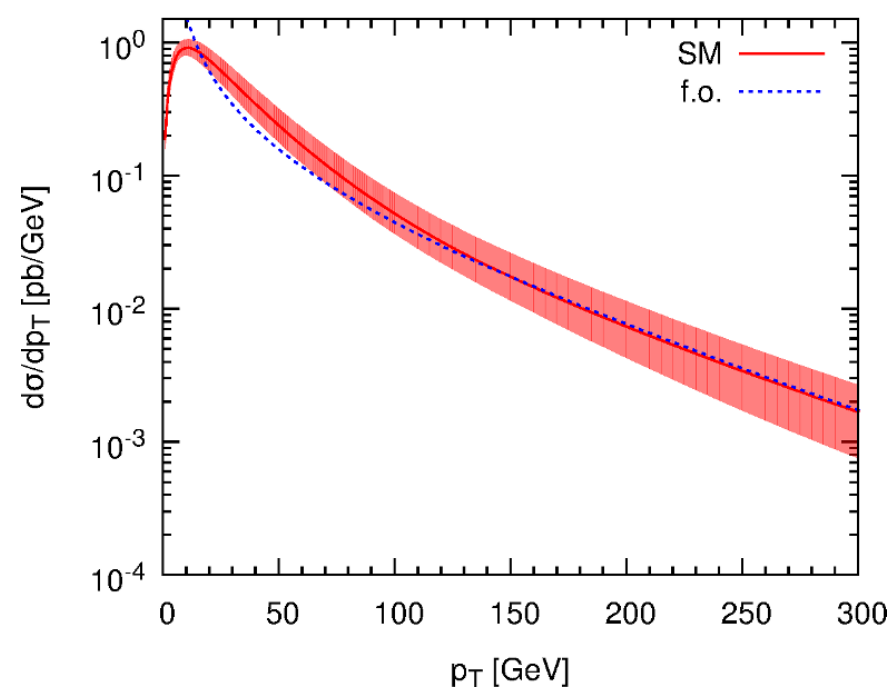

Figure 6. Transverse momentum distribution at NLO (blue, dotted) and NLO+NLL (red, solid) in the SM; lines: central scale choices; band: uncertainty on NLO+NLL due to scale variation as described in the text.

\section{Results}

We are now ready to present our results for the transverse momentum spectrum of MSSM Higgs bosons produced in gluon fusion through NLO+NLL.

For reference, figure 6 shows the $p_{T}$-spectrum of a SM Higgs boson of mass $m_{h}=$ $125.6 \mathrm{GeV}$. The resummed cross section is finite in the limit $p_{T} \rightarrow 0$ and smoothly matches the fixed order curve at large transverse momenta $\left(p_{T} \gtrsim m_{h}\right)$. The uncertainty band is obtained through scale variation of $\mu_{\mathrm{F}}, \mu_{\mathrm{R}}$ and $Q$, following the procedure described in section 4.2. In order to compare to other calculations, it may be useful to consider the ratio of the $p_{T}$ distribution which includes the full quark mass dependence to the result in the heavy-top limit (reweighted by the full LO inclusive cross section for $g g \rightarrow H$ ). The corresponding curve for the SM is shown in figure 7 and can be compared to analoguous plots of refs. [47, 50, 51, 100]. Disregarding the specific normalization in these papers, the behaviour of the curve which includes both top- and bottom-quark effects is quite different in the various approaches, in particular towards small values of $p_{T}$. For example, in ref. [51], where a common resummation scale for the top- and the bottom-quark effects of $Q_{0, t}=Q_{0, b}=Q_{0, \text { int }}=m_{\phi} / 2$ was chosen, the curve drops only by about $6 \%$ between $p_{T}=100 \mathrm{GeV}$ and $p_{T}=0$. With a separate resummation scale for the bottom-effects of $Q_{0, b}=Q_{0, \text { int }} \in\left[m_{\mathrm{b}}, 4 m_{\mathrm{b}}\right]$ as suggested in ref. [47], this effect becomes much more pronounced and amounts to about $(27 \pm 9) \%$. In the POWHEG approach of ref. [50], on the other hand, the drop in the curve is roughly 20\%, while the MC@NLO [101] result of ref. [100] with a drop of $5 \%$ is quite similar to the analytic resummation.

Separate resummation scales for the top, the bottom, and the interference term as given in table 1 , on the other hand, lead to a drop of $11 \%$, which is of a size as expected 


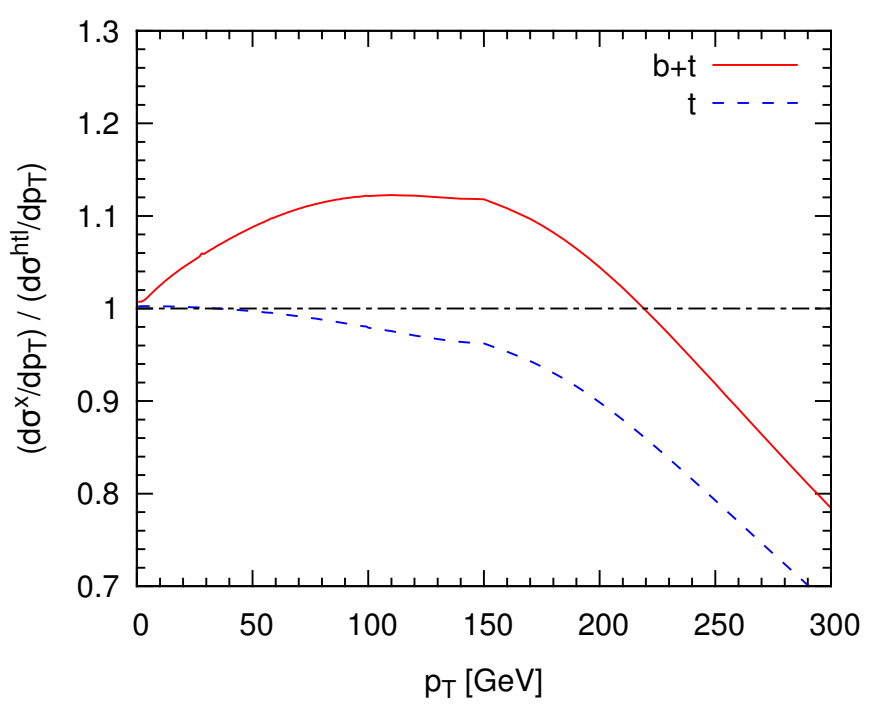

Figure 7. Exact top and bottom mass dependence of the transverse momentum distribution at NLO+NLL. In the dashed curve, bottom quark effects are set to zero. The normalization of these curves is to the respective LO total cross section times the shape of the NLO+NLL result in the heavy top limit.

considering the magnitude of the new scale choices compared to the ones of the previous studies in refs. [47, 51].

It has been shown that differences in the various approaches (analytic resummation, POWHEG, MC@NLO) become much smaller by a simultaneous adjustment of the corresponding intrinsic scales $\left(Q, h_{\text {fact }}\right.$, shower scale). Due to the similarities in their NLO matching, this leads to an excellent agreement for various scale choices [102] for the analytic resummation and MC@NLO. Also, the initially observed large differences to the POWHEG approach are alleviated [103] at least when in all approaches the scale for the bottom contribution is choosen of the order of the bottom mass.

The $p_{T}$ distributions of the light Higgs boson in the various scenarios of table 2 are virtually indistinguishable from the SM distribution shown in figure 6 . This is because the observation of a Higgs particle at about $m_{h}=125 \mathrm{GeV}$ typically constrains the parameter space of the MSSM in such a way that the light Higgs is SM-like.

In order to quantify the deviations between the MSSM and the SM prediction, figure 8 (a) shows the ratios

$$
R_{\mathcal{S}}\left(p_{T}\right)=\frac{\mathrm{d} \sigma_{\mathcal{S}} / \mathrm{d} p_{T}}{\mathrm{~d} \sigma_{\mathrm{SM}} / \mathrm{d} p_{T}}
$$

of the resummed $p_{T}$ distributions at NLO+NLL for the various MSSM scenarios $\mathcal{S}$ with respect to the SM one. The difference to the SM is typically at the 1-3\% level; only scenario $m_{h}^{\max }(300,6.5)$ deviates by up to $9 \%$. All curves are below one, because their respective total cross sections are smaller than the SM one. Considering the ratio for the shapes, i.e. 


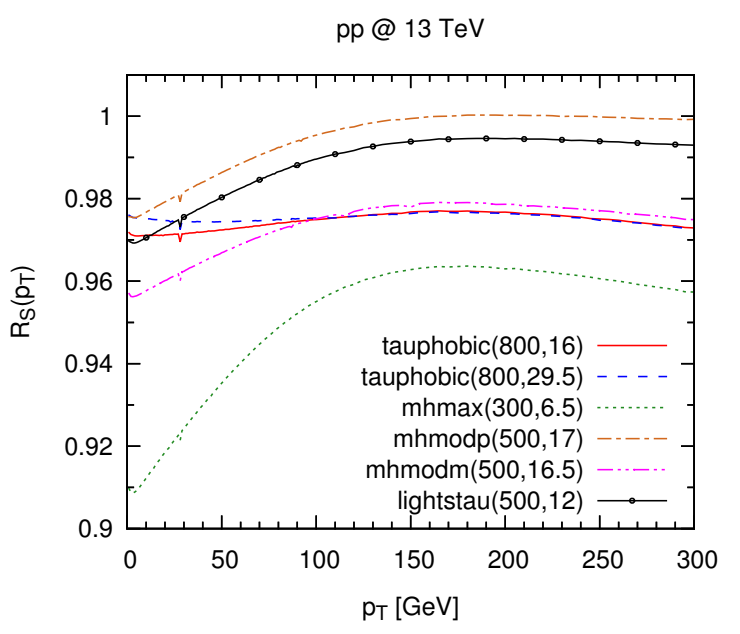

(a)

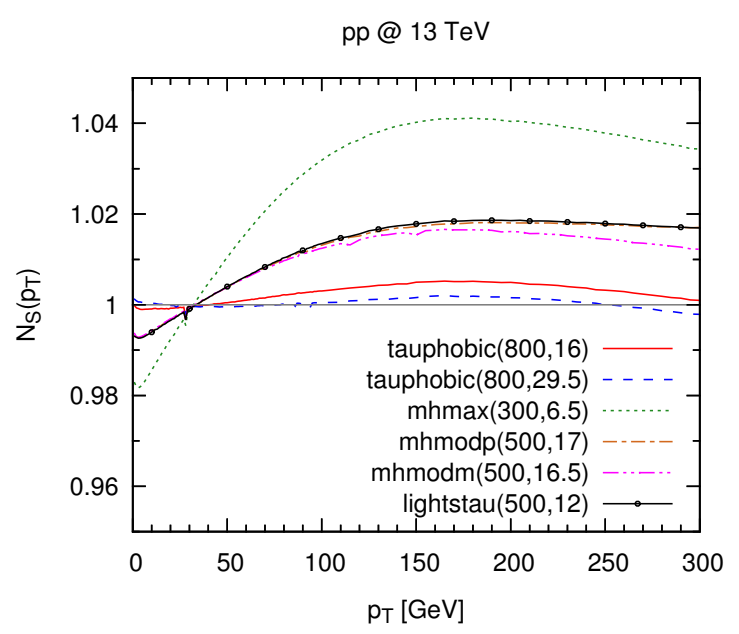

(b)

Figure 8. Resummed $p_{T}$ distributions in the various scenarios normalized to the SM distribution: (a) ratio $R(\mathcal{S})$ as defined in eq. (5.1) and (b) $N(\mathcal{S})$ as defined in eq. (5.2).

the normalized distributions, eq. (3.1),

$$
N_{\mathcal{S}}\left(p_{T}\right)=\frac{\mathrm{d} \bar{\sigma}_{\mathcal{S}} / \mathrm{d} p_{T}}{\mathrm{~d} \bar{\sigma}_{\mathrm{SM}} / \mathrm{d} p_{T}}
$$

we find variations at the $2 \%$-level, see figure $8(\mathrm{~b})$, with the only exception again $m_{h}^{\max }(300,6.5)$ which, however, still stays within $4 \%$ of the SM prediction. Apparently, the harder spectrum for the MSSM scenarios compared to the SM is due to a slightly larger negative int- $b$ term, recall figure 5 . The numerical effects observed here are roughly of the same size as those observed in ref. [50] (see the right plot of figure 8 in that paper). The fact that for all scenarios $\mathcal{S}$, the ratio $N_{\mathcal{S}}=1$ occurs at roughly the same value of $p_{T} \approx 30 \mathrm{GeV}$ is a consequence of the similar "barycenter" $\left\langle\hat{p}_{T}\right\rangle=\sigma_{\text {tot }}^{-1} \int \mathrm{d} p_{T} p_{T}\left(\mathrm{~d} \sigma / \mathrm{d} p_{T}\right)$ of the distributions.

While the predictions for the light Higgs are very SM-like, this is not the case for the heavy and pseudo-scalar Higgs, see figure 9. ${ }^{14}$ We show curves for various scenarios with $m_{A}=800 \mathrm{GeV}$, where $m_{H} \approx m_{A}$. Clearly, the absolute size of the cross section for both $H$ and $A$ depends strongly on the respective scenario and the value of $\tan \beta$ (see also ref. [82]). Indeed for the heavy Higgs, in all scenarios the cross section increases with the value of $\tan \beta$, which is caused by the fact that the bottom contribution strongly increases and eventually becomes the dominant contribution to the cross section. One remarkable observation is that for the pseudo-scalar Higgs the curves in the $\tau$-phobic scenarios for the two different values of $\tan \beta$ are quite close, in contrast to all other scenarios. In fact, at large values of $p_{T}$, the cross section for $\tan \beta=16$ is even bigger than the one for $\tan \beta=29.5$. The reason for this behavior will be discussed further below.

\footnotetext{
${ }^{14}$ In fact, note that, when the light Higgs is close to the decoupling limit, i.e. its couplings become identical to the SM ones, the opposite is the case for the heavy Higgs.
} 


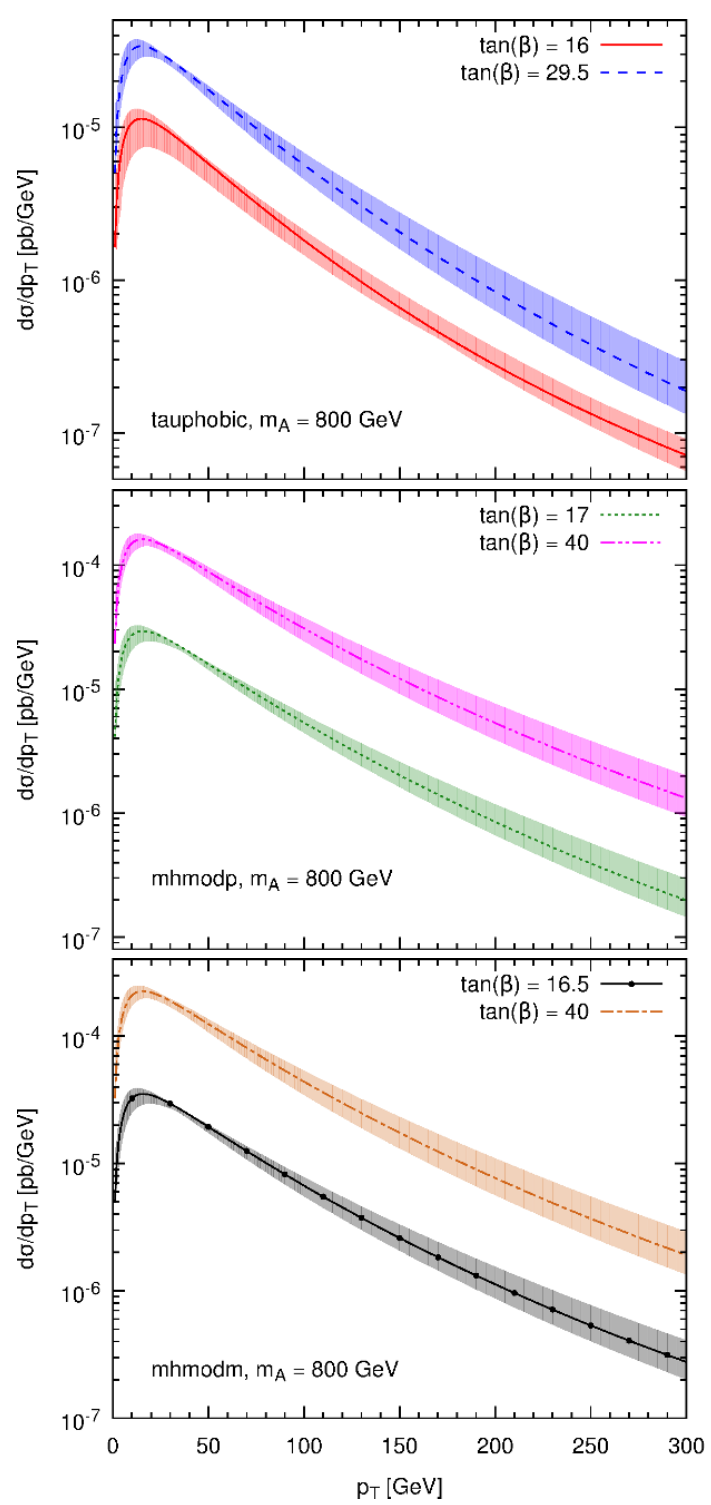

(a)

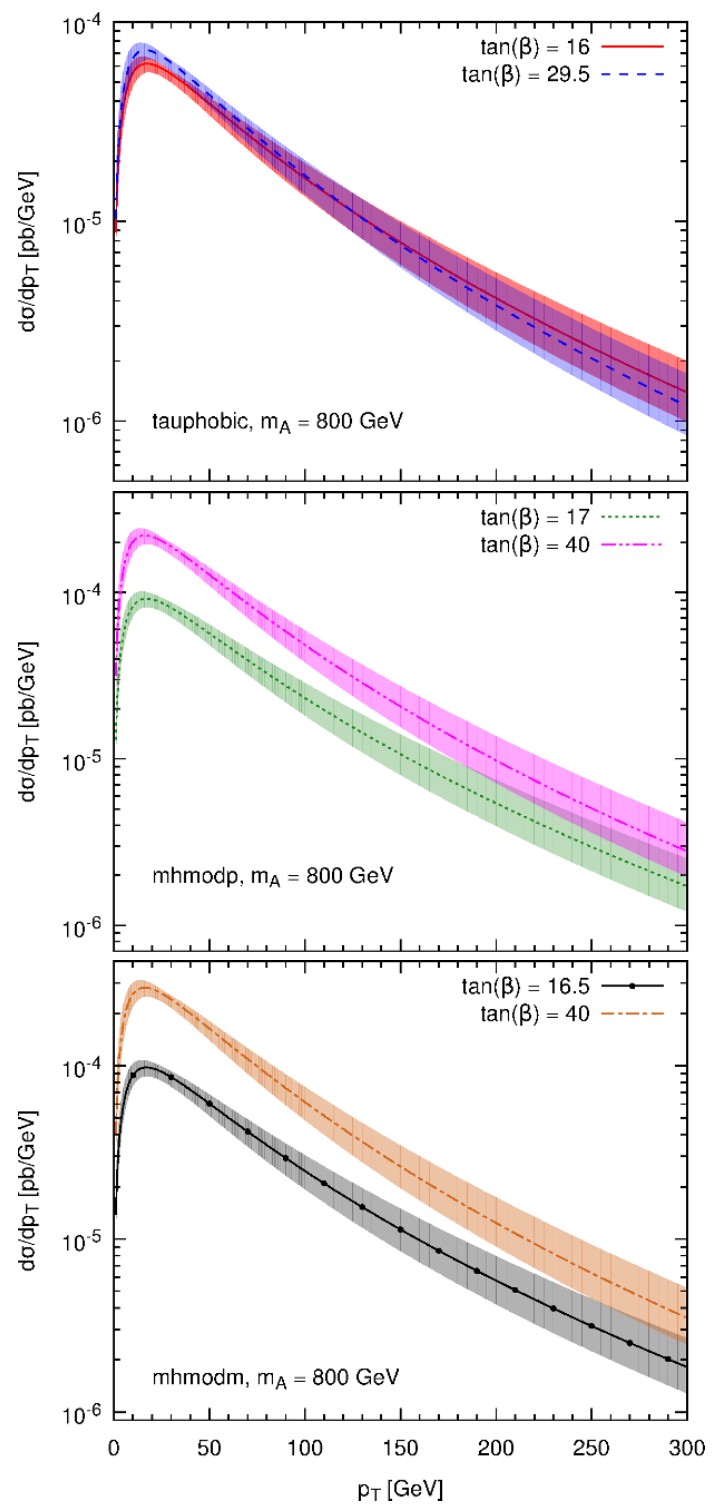

(b)

Figure 9. Transverse momentum distribution at NLO+NLL for (a) the heavy and (b) the pseudo-scalar MSSM Higgs boson for scenarios $\tau$-phobic $(800,16)$ (red, solid), $\tau$-phobic $(800,29.5)$ (blue, dashed), $m_{h}^{\bmod +}(800,17)$ (green, dotted), $m_{h}^{\bmod +}(800,40)$ (magenta, dash-double dotted), $m_{h}^{\bmod -}(800,16.5)$ (black, solid with dots) and $m_{h}^{\bmod -}(800,40)$ (brown, dash-dotted); lines: central scale choices; bands: uncertainty due to scale variation.

In section 3 , we split the cross section into the three contributions pure- $b$, no- $b$, and int- $b$ for which separate resummation scales were determined. The relative contribution of these three terms to the heavy Higgs $p_{T}$ distribution for (a) the $\tau$-phobic $(800,16)$ and (b) the $\tau$-phobic $(800,29.5)$ scenario is shown in figure 10 . Note that by definition the pure- $b$ (red, solid), no- $b$ (blue, dashed) and int- $b$ curve (brown, dash-dotted) add up to 


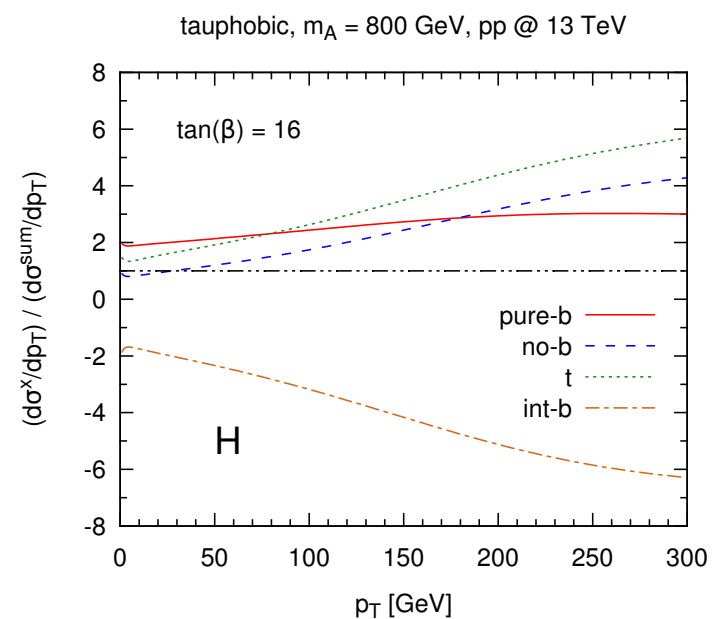

(a)

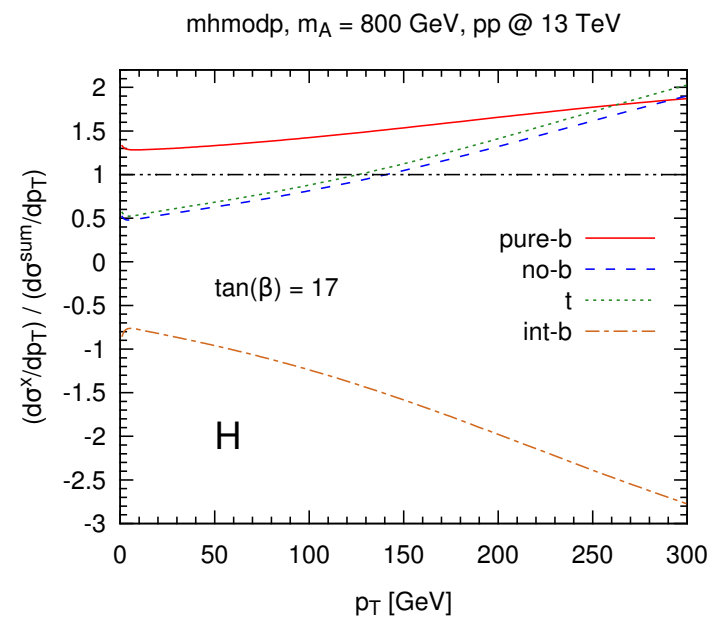

(c)

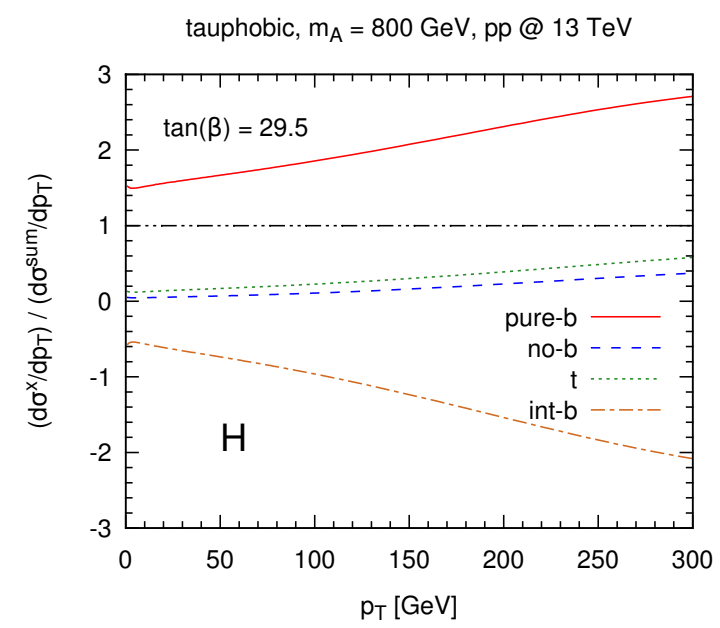

(b)

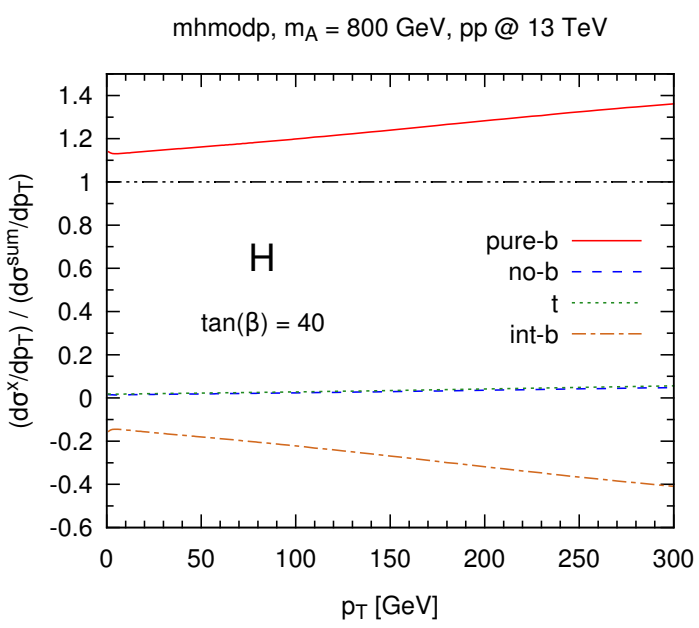

(d)

Figure 10. Relative contributions to the resummed $p_{T}$ distribution for the heavy Higgs normalized to the full cross section in (a) $\tau$-phobic $(800,16)$, (b) $\tau$-phobic $(800,29.5)$, (c) $m_{h}^{\bmod +}(800,17)$ and (d) $m_{h}^{\bmod +}(800,40)$. The pure bottom term (red, solid), the no- $b$ term (blue, dashed) and their interference (brown, dash-dotted) add up to one, which is marked for reference (black, dash-double dotted). For comparison, the pure top contribution is shown as well (green, dotted).

one (black, dash-double dotted). For comparison, we also include a curve for the "pure$t$ contribution" (green, dotted) which is defined to be proportional to the square of the top-Higgs coupling $y_{t}$.

For $\tau$-phobic $(800,16)$, we find a rather large cancellation between the positive no- $b$ and pure- $b$-, and the negative int- $b$ term, see figure 10 (a). It shows the importance of the proper treatment of the int- $b$ term in the resummation procedure and justifies a separate resummation scale as introduced in section 3. By comparing to the pure- $t$ contribution, we also observe that the squark effects are of the order of the overall contribution and therefore very relevant. 


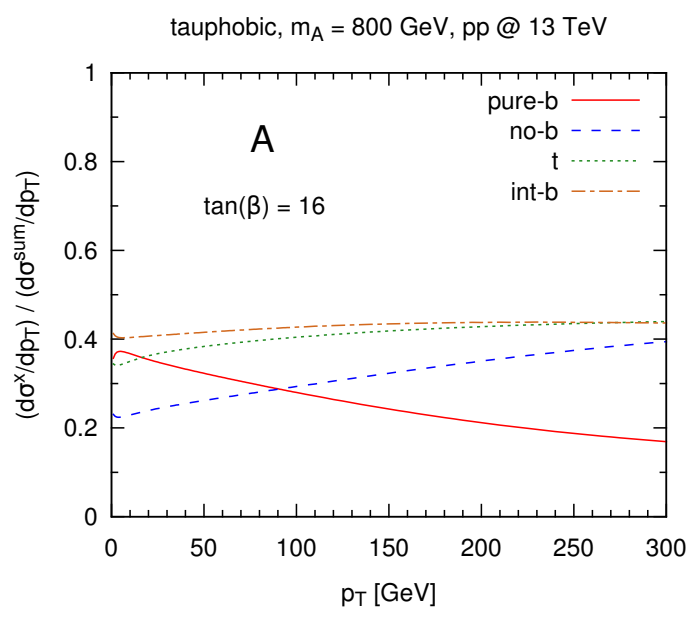

(a)

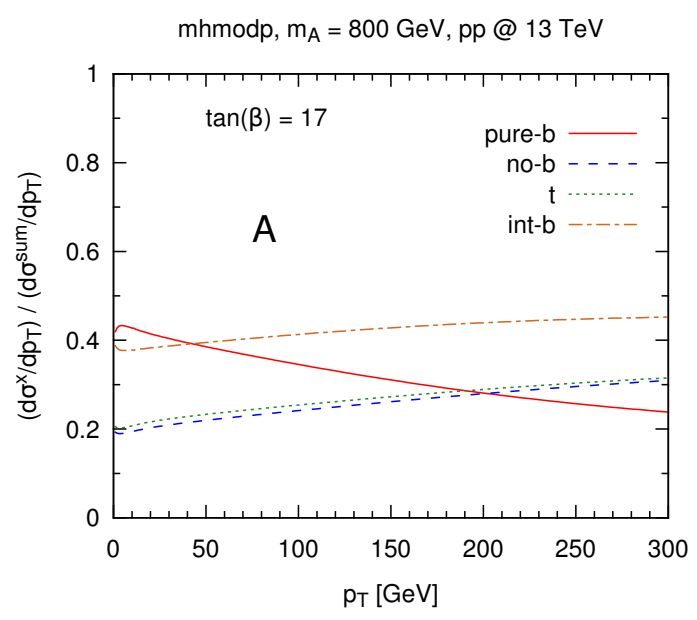

(c)

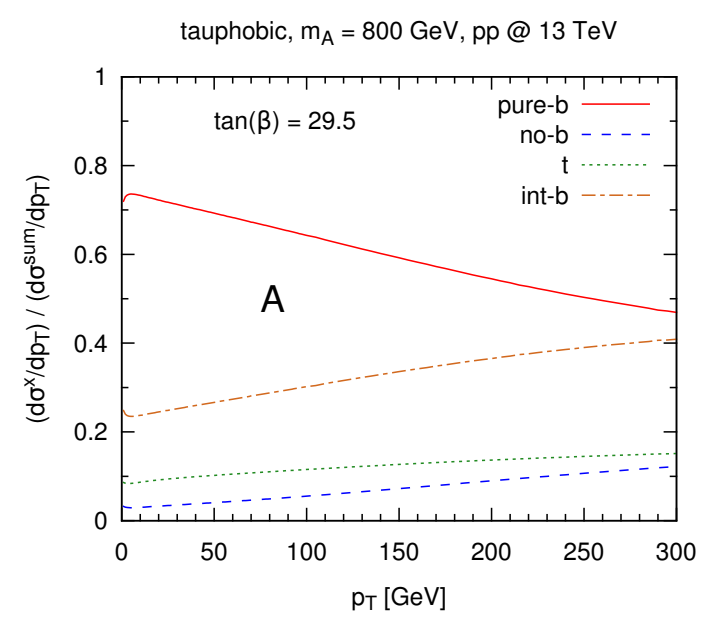

(b)

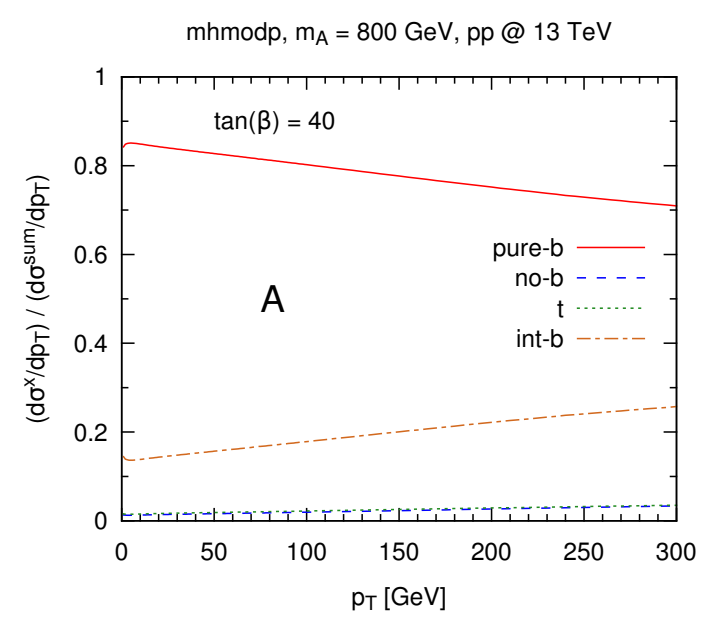

(d)

Figure 11. Same as figure 10, but for the pseudo-scalar Higgs.

Going to $\tan \beta=29.5$, the cancellations among the individual contributions are less severe, see figure 10 (b). As expected, the cross section is largely dominated by bottomquark effects, i.e., the pure- $b$ and the int- $b$ term. These results substantiate that the contribution of the bottom loop causes the increase of the cross section that we observed in figure 9 (a) at high $\tan \beta$.

Let us now compare these observations in the $\tau$-phobic to the $m_{h}^{\bmod +}$ scenario shown in figure $10(\mathrm{c})$ and (d) (the curves for the $m_{h}^{\bmod -}$ scenario are almost indistinguishable from $\left.m_{h}^{\bmod +}\right)$. The qualitative features of the plots in the two scenarios are quite similar. However, in the $m_{h}^{\bmod +}$ scenario, the cancellation between the int- $b$ - and the other terms is less pronounced. Also, the pure- $b$-contribution is typically more important than the no- $b$ one in this scenario, except for the large- $p_{T}$ region in the case $\tan \beta=17$. Squark effects are much smaller there, and at $\tan \beta=40$, the no-b-contribution is basically negligible over the full $p_{T}$ range. 


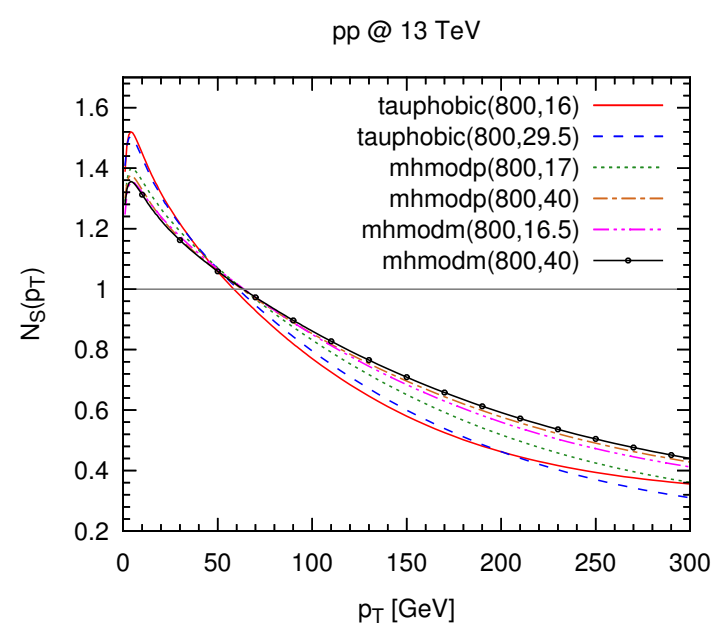

(a)

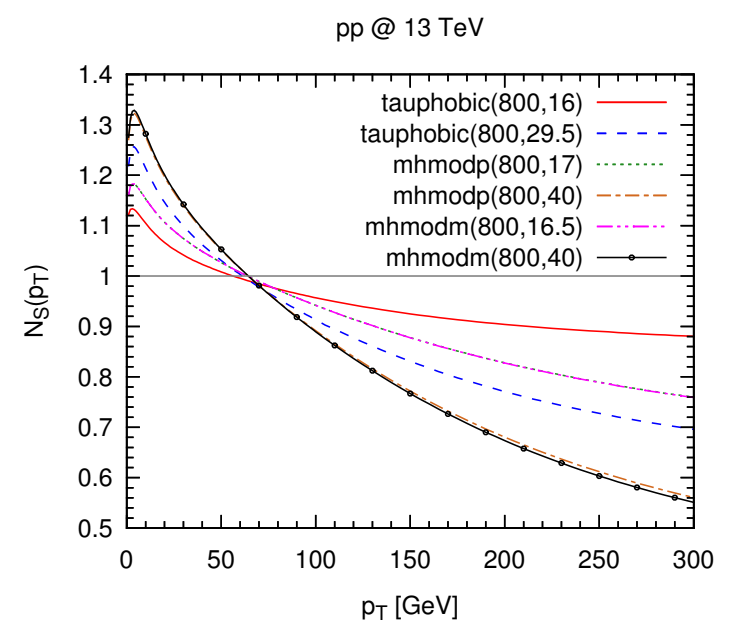

(b)

Figure 12. The ratio $N(\mathcal{S})$, defined in eq. $(5.2)$, of the resummed $p_{T}$ shapes in figure 9 for (a) the heavy Higgs and (b) the pseudo-scalar Higgs.

Figure 11 shows the same study for the pseudo-scalar Higgs (again, $m_{h}^{\bmod -}$ is almost identical to $m_{h}^{\text {mod+ }}$ ). The structure of the various contributions to the cross section is quite different from the one for the heavy Higgs. Since the int- $b$ term is positive here, all curves remain between 0 and 1 . In both scenarios, for the smaller value of $\tan \beta$, each term contributes at least about $20 \%$ to the cross section, and none of them exceeds $45 \%$. The largest contribution is due to the int- $b$ term for most transverse momenta. At high $\tan \beta$, the pure- $b$ contribution becomes clearly dominant again. While the int- $b$ term remains sizable, both pure- $t$ and no- $b$ terms are negligible, especially in the $m_{h}^{\bmod +}$ scenario.

With the results of figure 11 (a) and (b), it is interesting to take another look at the behavior of the distribution for the pseudo-scalar Higgs shown in figure 9 (b) in the $\tau$-phobic scenarios. The splitting into the individual contributions suggests that there is no deep reason for the similarity of the curves for $\tan \beta=16$ and $\tan \beta=29.5$. The hierarchy of the various contributions to the cross section in the $\tau$-phobic scenarios is not very different from the $m_{h}^{\text {mod+ }}$ scenarios of figure 11. It rather seems to be an accidental interplay of the top- and bottom-quark effects so that the absolute size of the increase of the pure- $b$ contribution from $\tan \beta=16$ to $\tan \beta=29.5$ is compensated by a decrease of similar size of the no- $b$ and int- $b$ term.

To finalize the analysis of the $p_{T}$ distributions shown in figure 9 , we study their shapes in the various scenarios for the heavy Higgs in figure 12 (a) and for the pseudo-scalar Higgs in figure 12 (b) by considering again the ratio of shapes defined in eq. (5.2). Note that the normalization is for a "SM Higgs" of mass $800 \mathrm{GeV}$. For the heavy Higgs in figure 12 (a), we observe generally small deviations between the curves. While the biggest difference occurs at large transverse momenta, the similarity in shape at small $p_{T}$ is remarkable. Their deviation from the SM curve is quite large though, reaching up to $60 \%$, and clearly showing the dominance of the pure- $b$ term by the significantly softer spectrum, see figure 5 . For most scenarios, however, the softness decreases with increasing $\tan \beta$, with the exception of the $\tau$-phobic scenarios. This is again the impact of the negative int- $b$ term. The softening 
mhmax, pp@13 TeV

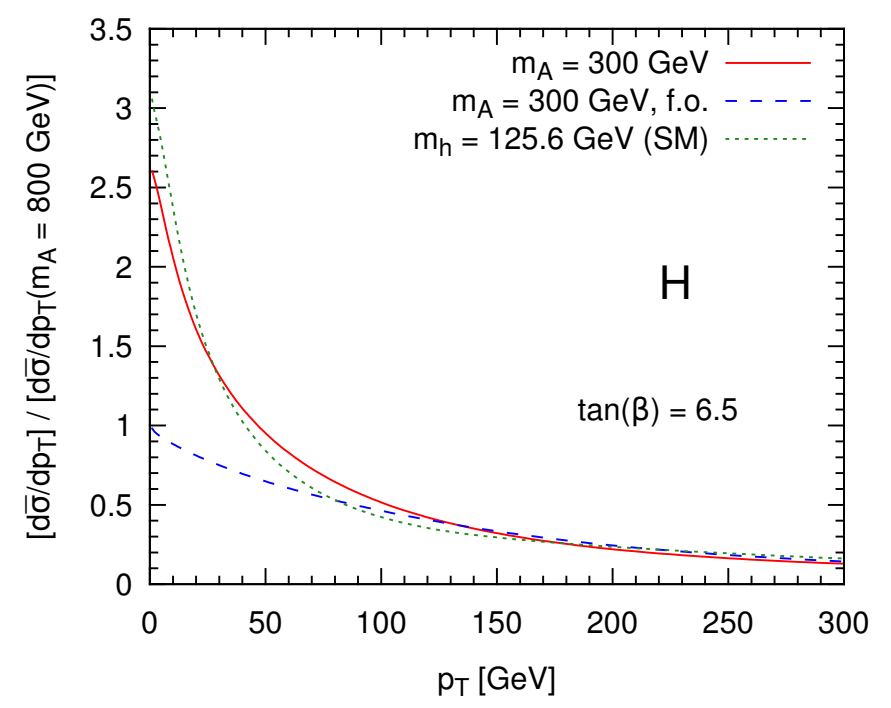

Figure 13. Ratio of resummed $p_{T}$ shapes in the $m_{h}^{\max }$ scenario at $m_{H}=300 \mathrm{GeV}$ and $m_{H}=$ $800 \mathrm{GeV}$ (red, solid) for the heavy Higgs, the corresponding fixed order curve (blue, dashed) and, for comparison, the ratio of the resummed distribution of the light Higgs at $m_{h}=125.6 \mathrm{GeV}$ and the heavy Higgs at $m_{H}=800 \mathrm{GeV}$ (green, dotted).

of the spectrum due to an enhanced $b$-contribution is in agreement with the observations of refs. [50,75].

Considering the pseudo-scalar Higgs in figure $12(\mathrm{~b})$, the spread of the curves is significantly larger both at small and high transverse momenta, leading to a more enhanced difference in shape of the resummed $p_{T}$ distributions in the various scenarios. Since the interference contributions are strictly positive in this case, the deviation from the SM increases with increasing $\tan \beta$ for all scenarios, including $\tau$-phobic. We also note that the $m_{h}^{\text {mod }+}$ and $m_{h}^{\text {mod- }}$ curves are practically indistinguishable in this case.

As a final study, we compare the transverse momentum distributions at different Higgs masses. Figure 13 shows the ratio of the shapes for the heavy scalar in the $m_{h}^{\max }(300,6.5)$ and in the $m_{h}^{\max }(800,6.5)$ scenario. For comparison, the same ratio is shown at fixed order (blue, dashed). In addition, the ratio of the $p_{T}$-shape of a SM Higgs at $125.6 \mathrm{GeV}$ and the heavy Higgs in the $m_{h}^{\max }(800,6.5)$ scenario (green, dotted) is given. The spectra at low Higgs masses are significantly softer due to increased soft radiation. This observation is consistent with the behavior of figures 6 and 9 (a), which differ by an order of magnitude in the difference between the minimum at $p_{T}=300 \mathrm{GeV}$ and the maximum of the curve. Furthermore, figure 13 confirms that the shape at high transverse momenta is driven by the fixed order cross section, as expected. The harder spectrum at high Higgs masses is caused by the fact that the colliding gluons carry more energy in a production of a heavy particle, which makes it more likely to emit harder gluons and therefore, to produce a harder Higgs boson. 


\section{Conclusions}

We have presented typical MSSM effects on the $p_{T}$ spectrum of a neutral Higgs boson produced at the LHC. Special emphasis has been put on the impact of bottom quarks. While the current experimental data imply a small, SM-like bottom-Yukawa coupling for the light Higgs boson, the production mechanism for the heavy and the pseudo-scalar Higgs can be dominated by bottom-quark loops.

Through a pragmatic argumentation based on simple theoretical and physical expectations, we derived separate resummation scales for the pure- $b$ and int- $b$ contributions which turn out to be significantly larger than the bottom-quark mass, and smaller than the Higgs boson mass.

We find the well-known behavior that the bottom loop typically softens the $p_{T^{-}}$ spectrum. For the pseudo-scalar Higgs boson, the int- $b$ term is typically positive and leads to a further softening of the spectrum. For the light and heavy CP-even Higgs bosons, on the other hand, the int- $b$ term is typically negative, and makes the spectrum a bit harder. The latter effect is specifically visible for the light Higgs where the spectrum in all MSSM benchmarks is harder than in the SM, because the relative contribution of the negative int- $b$ term is larger. In all scenarios, we observe an enhanced importance of the bottom-quark contributions for the heavy and pseudo-scalar Higgs, which become by far dominant at large values of $\tan \beta$. Indeed, the corresponding spectra are clearly softer than in the SM. Finally, we confirmed that larger Higgs masses lead to reduced soft gluon emission and therefore a harder spectrum.

The resummed $p_{T}$-distributions through NLO+NLL have been implemented in the program MoRe-SusHi, which advances the program SusHi to small- $p_{T}$ distributions. The code is publically available and can be found on the SusHi homepage. ${ }^{15}$

As an outlook, one may consider combining the consistent NLO+NLL results presented in this paper for the $p_{T}$-distribution of MSSM Higgs bosons with the NNLO+NNLL distribution in the infinite-top mass approximation, and possibly even with the $p_{T}$ spectrum of the Higgs boson produced through bottom-quark annihilation. This is certainly beyond the scope of this paper and is left for a future publication.

\section{Acknowledgments}

We are indebted to Stefan Liebler for comments on the manuscript, for suggesting the name "MoRe-SusHi", and for letting us use the diagrams in figure 1. We would like to thank Alessandro Vicini for helpful communication and comments on the manuscript. MW would like to thank Pier Francesco Monni for several fruitful discussions. This research was supported by the Munich Institute for Astro- and Particle Physics (MIAPP) of the DFG cluster of excellence "Origin and Structure of the Universe" and the BMBF, contract 05H12PXE. HM was supported by a Marie Curie Early Initial Training Network Fellowship of the European Community's Seventh Framework Programme under contract number (PITNGA-2012-315877-MCnetITN). MW was supported by the European Commission through the FP7 Marie Curie Initial Training Network "LHCPhenoNet" (PITN-GA-2010-264564).

\footnotetext{
${ }^{15}$ http://sushi.hepforge.org/moresushi
} 


\section{A $\quad g^{(n)}$-functions at NLL}

The Sudakov form factor can be expressed in terms of functions $g^{(n)}$, when the argument of the exponential is expanded with respect to $\alpha_{s}$, while treating $\alpha_{s} L$ as being of order unity, see eq. (2.7). To achieve NLL accuracy the first two functions have to be taken into account. Their functional expressions for $Q=\mu_{\mathrm{R}}=M$ are the following:

$$
\begin{aligned}
g^{(1)}\left(\alpha_{s} L\right)= & \frac{A_{c}^{(1)}}{\beta_{0}} \frac{\lambda+\ln (1-\lambda)}{\lambda}, \\
g^{(2)}\left(\alpha_{s} L\right)= & \frac{B_{c}^{(1)}}{\beta_{0}} \ln (1-\lambda)-\frac{A_{c}^{(2)}}{\beta_{0}^{2}}\left(\ln (1-\lambda)+\frac{\lambda}{1-\lambda}\right) \\
& +\frac{A_{c}^{(1)} \beta_{1}}{\beta_{0}^{3}}\left(\frac{1}{2} \ln ^{2}(1-\lambda)+\frac{\lambda+\ln (1-\lambda)}{1-\lambda}\right),
\end{aligned}
$$

where

$$
\lambda=\beta_{0} \frac{\alpha_{s}(M)}{\pi} L
$$

and $\beta_{0}=\left(11 C_{A}-2 N_{f}\right) / 12$ and $\beta_{1}=\left(17 C_{A}^{2}-5 C_{A} N_{f}-3 C_{F} N_{f}\right) / 24$ are the first two coefficients of the $\beta$ function.

The process and resummation scheme independent coefficients needed at NLL have been known for some time for gluon-induced processes [40]; they read

$$
\begin{aligned}
A_{g}^{(1)} & =C_{A}, \quad A_{g}^{(2)}=\frac{1}{2} C_{A}\left[\left(\frac{67}{18}-\frac{\pi^{2}}{6}\right) C_{A}-\frac{5}{9} N_{f}\right], \\
B_{g}^{(1)} & =-\beta_{0}=-\left(\frac{11}{6} C_{A}+\frac{1}{3} N_{f}\right),
\end{aligned}
$$

where $C_{F}=4 / 3, C_{A}=3$, and $N_{f}=5$ is the number of active quark flavors.

Open Access. This article is distributed under the terms of the Creative Commons Attribution License (CC-BY 4.0), which permits any use, distribution and reproduction in any medium, provided the original author(s) and source are credited.

\section{References}

[1] ATLAS collaboration, Observation of a new particle in the search for the standard model Higgs boson with the ATLAS detector at the LHC, Phys. Lett. B 716 (2012) 1 [arXiv:1207.7214] [INSPIRE].

[2] CMS collaboration, Observation of a new boson at a mass of $125 \mathrm{GeV}$ with the CMS experiment at the LHC, Phys. Lett. B 716 (2012) 30 [arXiv:1207.7235] [INSPIRE].

[3] LhC Higgs Cross Section Working Group collaboration, S. Dittmaier et al., Handbook of LHC Higgs cross sections: 1. Inclusive observables, arXiv:1101.0593 [INSPIRE].

[4] S. Dittmaier et al., Handbook of LHC Higgs cross sections: 2. Differential distributions, arXiv: 1201.3084 [INSPIRE]. 
[5] LhC Higgs Cross Section Working Group collaboration, S. Heinemeyer et al., Handbook of LHC Higgs cross sections: 3. Higgs properties, arXiv:1307.1347 [INSPIRE].

[6] R.V. Harlander and W.B. Kilgore, Next-to-next-to-leading order Higgs production at hadron colliders, Phys. Rev. Lett. 88 (2002) 201801 [hep-ph/0201206] [INSPIRE].

[7] C. Anastasiou and K. Melnikov, Higgs boson production at hadron colliders in NNLO QCD, Nucl. Phys. B 646 (2002) 220 [hep-ph/0207004] [INSPIRE].

[8] V. Ravindran, J. Smith and W.L. van Neerven, NNLO corrections to the total cross-section for Higgs boson production in hadron hadron collisions, Nucl. Phys. B 665 (2003) 325 [hep-ph/0302135] [INSPIRE].

[9] R.D. Ball, M. Bonvini, S. Forte, S. Marzani and G. Ridolfi, Higgs production in gluon fusion beyond NNLO, Nucl. Phys. B 874 (2013) 746 [arXiv:1303.3590] [INSPIRE].

[10] S. Buehler and A. Lazopoulos, Scale dependence and collinear subtraction terms for Higgs production in gluon fusion at N3LO, JHEP 10 (2013) 096 [arXiv: 1306.2223] [INSPIRE].

[11] R. Boughezal, F. Caola, K. Melnikov, F. Petriello and M. Schulze, Higgs boson production in association with a jet at next-to-next-to-leading order in perturbative QCD, JHEP 06 (2013) 072 [arXiv: 1302.6216] [inSPIRE].

[12] C. Anastasiou et al., Higgs boson gluon-fusion production at threshold in $N^{3} L O Q C D$, Phys. Lett. B 737 (2014) 325 [arXiv:1403.4616] [INSPIRE].

[13] S. Catani, D. de Florian, M. Grazzini and P. Nason, Soft gluon resummation for Higgs boson production at hadron colliders, JHEP 07 (2003) 028 [hep-ph/0306211] [INSPIRE].

[14] A. Idilbi, X.-d. Ji, J.-P. Ma and F. Yuan, Threshold resummation for Higgs production in effective field theory, Phys. Rev. D 73 (2006) 077501 [hep-ph/0509294] [INSPIRE].

[15] V. Ravindran, Higher-order threshold effects to inclusive processes in QCD, Nucl. Phys. B 752 (2006) 173 [hep-ph/0603041] [INSPIRE].

[16] V. Ahrens, T. Becher, M. Neubert and L.L. Yang, Renormalization-group improved prediction for Higgs production at hadron colliders, Eur. Phys. J. C 62 (2009) 333 [arXiv: 0809.4283] [INSPIRE].

[17] A. Djouadi and P. Gambino, Leading electroweak correction to Higgs boson production at proton colliders, Phys. Rev. Lett. 73 (1994) 2528 [hep-ph/9406432] [INSPIRE].

[18] G. Degrassi and F. Maltoni, Two-loop electroweak corrections to Higgs production at hadron colliders, Phys. Lett. B 600 (2004) 255 [hep-ph/0407249] [INSPIRE].

[19] U. Aglietti, R. Bonciani, G. Degrassi and A. Vicini, Two loop light fermion contribution to Higgs production and decays, Phys. Lett. B 595 (2004) 432 [hep-ph/0404071] [InSPIRE].

[20] S. Actis, G. Passarino, C. Sturm and S. Uccirati, NLO electroweak corrections to Higgs boson production at hadron colliders, Phys. Lett. B 670 (2008) 12 [arXiv:0809.1301] [INSPIRE].

[21] C. Anastasiou, R. Boughezal and F. Petriello, Mixed QCD-electroweak corrections to Higgs boson production in gluon fusion, JHEP 04 (2009) 003 [arXiv: 0811.3458] [INSPIRE].

[22] S. Marzani, R.D. Ball, V. Del Duca, S. Forte and A. Vicini, Higgs production via gluon-gluon fusion with finite top mass beyond next-to-leading order, Nucl. Phys. B 800 (2008) 127 [arXiv: 0801.2544] [INSPIRE].

[23] R.V. Harlander and K.J. Ozeren, Finite top mass effects for hadronic Higgs production at next-to-next-to-leading order, JHEP 11 (2009) 088 [arXiv:0909.3420] [INSPIRE]. 
[24] R.V. Harlander, H. Mantler, S. Marzani and K.J. Ozeren, Higgs production in gluon fusion at next-to-next-to-leading order QCD for finite top mass, Eur. Phys. J. C 66 (2010) 359 [arXiv:0912.2104] [INSPIRE].

[25] A. Pak, M. Rogal and M. Steinhauser, Finite top quark mass effects in NNLO Higgs boson production at LHC, JHEP 02 (2010) 025 [arXiv: 0911.4662] [INSPIRE].

[26] A. Pak, M. Rogal and M. Steinhauser, Production of scalar and pseudo-scalar Higgs bosons to next-to-next-to-leading order at hadron colliders, JHEP 09 (2011) 088 [arXiv:1107.3391] [INSPIRE].

[27] M. Spira, A. Djouadi, D. Graudenz and P.M. Zerwas, Higgs boson production at the LHC, Nucl. Phys. B 453 (1995) 17 [hep-ph/9504378] [INSPIRE].

[28] R. Harlander, Supersymmetric Higgs production at the Large Hadron Collider, Eur. Phys. J. C 33 (2004) S454 [hep-ph/0311005] [INSPIRE].

[29] R.V. Harlander and T. Neumann, Probing the nature of the Higgs-gluon coupling, Phys. Rev. D 88 (2013) 074015 [arXiv: 1308.2225] [InSPIRE].

[30] C. Grojean, E. Salvioni, M. Schlaffer and A. Weiler, Very boosted Higgs in gluon fusion, JHEP 05 (2014) 022 [arXiv: 1312.3317] [InSPIRE].

[31] A. Azatov and A. Paul, Probing Higgs couplings with high $p_{T}$ Higgs production, JHEP 01 (2014) 014 [arXiv: 1309.5273] [INSPIRE].

[32] D. de Florian, M. Grazzini and Z. Kunszt, Higgs production with large transverse momentum in hadronic collisions at next-to-leading order, Phys. Rev. Lett. 82 (1999) 5209 [hep-ph/9902483] [INSPIRE].

[33] V. Ravindran, J. Smith and W.L. Van Neerven, Next-to-leading order QCD corrections to differential distributions of Higgs boson production in hadron hadron collisions, Nucl. Phys. B 634 (2002) 247 [hep-ph/0201114] [INSPIRE].

[34] C.J. Glosser and C.R. Schmidt, Next-to-leading corrections to the Higgs boson transverse momentum spectrum in gluon fusion, JHEP 12 (2002) 016 [hep-ph/0209248] [INSPIRE].

[35] R.V. Harlander, T. Neumann, K.J. Ozeren and M. Wiesemann, Top-mass effects in differential Higgs production through gluon fusion at order $\alpha_{s}^{4}$, JHEP 08 (2012) 139 [arXiv:1206.0157] [INSPIRE].

[36] T. Neumann and M. Wiesemann, Finite top-mass effects in gluon-induced Higgs production with a jet-veto at $N N L O$, arXiv:1408.6836 [INSPIRE].

[37] C. Anastasiou, K. Melnikov and F. Petriello, Higgs boson production at hadron colliders: differential cross sections through next-to-next-to-leading order, Phys. Rev. Lett. 93 (2004) 262002 [hep-ph/0409088] [INSPIRE].

[38] S. Catani and M. Grazzini, An NNLO subtraction formalism in hadron collisions and its application to Higgs boson production at the LHC, Phys. Rev. Lett. 98 (2007) 222002 [hep-ph/0703012] [INSPIRE].

[39] S. Catani and M. Grazzini, HNNLO: a Monte Carlo program to compute Higgs boson production at hadron colliders, PoS (RAD COR 2007) 046 [arXiv:0802.1410] [INSPIRE].

[40] S. Catani, E. D'Emilio and L. Trentadue, The gluon form-factor to higher orders: gluon gluon annihilation at small Q-transverse, Phys. Lett. B 211 (1988) 335 [INSPIRE].

[41] C.P. Yuan, Kinematics of the Higgs boson at hadron colliders: NLO QCD gluon resummation, Phys. Lett. B 283 (1992) 395 [INSPIRE].

[42] R.P. Kauffman, Higher order corrections to Higgs boson $p_{T}$, Phys. Rev. D 45 (1992) 1512 [INSPIRE]. 
[43] G. Bozzi, S. Catani, D. de Florian and M. Grazzini, Transverse-momentum resummation and the spectrum of the Higgs boson at the LHC, Nucl. Phys. B 737 (2006) 73 [hep-ph/0508068] [INSPIRE].

[44] G. Bozzi, S. Catani, D. de Florian and M. Grazzini, The $q(T)$ spectrum of the Higgs boson at the LHC in QCD perturbation theory, Phys. Lett. B 564 (2003) 65 [hep-ph/0302104] [INSPIRE].

[45] D. de Florian, G. Ferrera, M. Grazzini and D. Tommasini, Transverse-momentum resummation: Higgs boson production at the Tevatron and the LHC, JHEP 11 (2011) 064 [arXiv:1109.2109] [INSPIRE].

[46] D. de Florian, G. Ferrera, M. Grazzini and D. Tommasini, Higgs boson production at the LHC: transverse momentum resummation effects in the $H \rightarrow 2 \gamma, H \rightarrow W W \rightarrow l \nu l \nu$ and $H \rightarrow Z Z \rightarrow 4 l$ decay modes, JHEP 06 (2012) 132 [arXiv:1203.6321] [INSPIRE].

[47] M. Grazzini and H. Sargsyan, Heavy-quark mass effects in Higgs boson production at the LHC, JHEP 09 (2013) 129 [arXiv:1306.4581] [INSPIRE].

[48] P. Nason, A new method for combining NLO QCD with shower Monte Carlo algorithms, JHEP 11 (2004) 040 [hep-ph/0409146] [INSPIRE].

[49] S. Alioli, P. Nason, C. Oleari and E. Re, NLO vector-boson production matched with shower in POWHEG, JHEP 07 (2008) 060 [arXiv:0805.4802] [INSPIRE].

[50] E. Bagnaschi, G. Degrassi, P. Slavich and A. Vicini, Higgs production via gluon fusion in the POWHEG approach in the SM and in the MSSM, JHEP 02 (2012) 088 [arXiv:1111.2854] [INSPIRE].

[51] H. Mantler and M. Wiesemann, Top-and bottom-mass effects in hadronic Higgs production at small transverse momenta through LO+NLL, Eur. Phys. J. C 73 (2013) 2467 [arXiv: 1210.8263] [INSPIRE].

[52] A. Banfi, P.F. Monni and G. Zanderighi, Quark masses in Higgs production with a jet veto, JHEP 01 (2014) 097 [arXiv:1308.4634] [InSPIRE].

[53] R.V. Harlander, A. Tripathi and M. Wiesemann, Higgs production in bottom quark annihilation: transverse momentum distribution at NNLO+NNLL, Phys. Rev. D 90 (2014) 015017 [arXiv: 1403.7196] [INSPIRE].

[54] R.V. Harlander, K.J. Ozeren and M. Wiesemann, Higgs plus jet production in bottom quark annihilation at next-to-leading order, Phys. Lett. B 693 (2010) 269 [arXiv:1007.5411] [INSPIRE].

[55] K.J. Ozeren, Analytic results for Higgs production in bottom fusion, JHEP 11 (2010) 084 [arXiv:1010.2977] [INSPIRE].

[56] R. Harlander and M. Wiesemann, Jet-veto in bottom-quark induced Higgs production at next-to-next-to-leading order, JHEP 04 (2012) 066 [arXiv:1111.2182] [INSPIRE].

[57] S. Bühler, F. Herzog, A. Lazopoulos and R. Müller, The fully differential hadronic production of a Higgs boson via bottom quark fusion at NNLO, JHEP 07 (2012) 115 [arXiv:1204.4415] [INSPIRE].

[58] R.V. Harlander and M. Steinhauser, Hadronic Higgs production and decay in supersymmetry at next-to-leading order, Phys. Lett. B 574 (2003) 258 [hep-ph/0307346] [INSPIRE].

[59] R.V. Harlander and M. Steinhauser, Supersymmetric Higgs production in gluon fusion at next-to-leading order, JHEP 09 (2004) 066 [hep-ph/0409010] [INSPIRE].

[60] R.V. Harlander and F. Hofmann, Pseudo-scalar Higgs production at next-to-leading order SUSY-QCD, JHEP 03 (2006) 050 [hep-ph/0507041] [INSPIRE]. 
[61] M. Mühlleitner and M. Spira, Higgs boson production via gluon fusion: squark loops at NLO QCD, Nucl. Phys. B 790 (2008) 1 [hep-ph/0612254] [INSPIRE].

[62] G. Degrassi and P. Slavich, On the NLO QCD corrections to Higgs production and decay in the MSSM, Nucl. Phys. B 805 (2008) 267 [arXiv:0806.1495] [InSPIRE].

[63] C. Anastasiou, S. Beerli and A. Daleo, The two-loop QCD amplitude gg $\rightarrow h, H$ in the minimal supersymmetric standard model, Phys. Rev. Lett. 100 (2008) 241806 [arXiv:0803.3065] [INSPIRE].

[64] R.V. Harlander, F. Hofmann and H. Mantler, Supersymmetric Higgs production in gluon fusion, JHEP 02 (2011) 055 [arXiv: 1012.3361] [INSPIRE].

[65] G. Degrassi and P. Slavich, NLO QCD bottom corrections to Higgs boson production in the MSSM, JHEP 11 (2010) 044 [arXiv: 1007.3465] [INSPIRE].

[66] M. Muhlleitner, H. Rzehak and M. Spira, SUSY-QCD corrections to MSSM Higgs boson production via gluon fusion, PoS (RADCOR2009) 043 [arXiv: 1001.3214] [INSPIRE].

[67] G. Degrassi, S. Di Vita and P. Slavich, NLO QCD corrections to pseudoscalar Higgs production in the MSSM, JHEP 08 (2011) 128 [arXiv:1107.0914] [INSPIRE].

[68] G. Degrassi, S. Di Vita and P. Slavich, On the NLO QCD corrections to the production of the heaviest neutral Higgs scalar in the MSSM, Eur. Phys. J. C 72 (2012) 2032 [arXiv:1204.1016] [INSPIRE].

[69] R.V. Harlander, S. Liebler and H. Mantler, SusHi: a program for the calculation of Higgs production in gluon fusion and bottom-quark annihilation in the Standard Model and the MSSM, Computer Physics Communications 184 (2013) 1605 [arXiv:1212.3249] [INSPIRE].

[70] R. Harlander, M. Mühlleitner, J. Rathsman, M. Spira and O. Stål, Interim recommendations for the evaluation of Higgs production cross sections and branching ratios at the LHC in the Two-Higgs-Doublet Model, arXiv:1312.5571 [INSPIRE].

[71] S. Catani and M. Grazzini, QCD transverse-momentum resummation in gluon fusion processes, Nucl. Phys. B 845 (2011) 297 [arXiv:1011.3918] [InSPIRE].

[72] J.C. Collins, D.E. Soper and G.F. Sterman, Transverse momentum distribution in Drell-Yan pair and $W$ and $Z$ boson production, Nucl. Phys. B 250 (1985) 199 [INSPIRE].

[73] S. Catani, D. de Florian and M. Grazzini, Universality of nonleading logarithmic contributions in transverse momentum distributions, Nucl. Phys. B 596 (2001) 299 [hep-ph/0008184] [INSPIRE].

[74] D. de Florian and M. Grazzini, The structure of large logarithmic corrections at small transverse momentum in hadronic collisions, Nucl. Phys. B 616 (2001) 247 [hep-ph/0108273] [INSPIRE].

[75] U. Langenegger, M. Spira, A. Starodumov and P. Trueb, SM and MSSM Higgs boson production: spectra at large transverse momentum, JHEP 06 (2006) 035 [hep-ph/0604156] [INSPIRE].

[76] A.D. Martin, W.J. Stirling, R.S. Thorne and G. Watt, Parton distributions for the LHC, Eur. Phys. J. C 63 (2009) 189 [arXiv:0901.0002] [InSPIRE].

[77] M.S. Carena, D. Garcia, U. Nierste and C.E.M. Wagner, Effective Lagrangian for the $\bar{t} b H^{+}$ interaction in the MSSM and charged Higgs phenomenology, Nucl. Phys. B 577 (2000) 88 [hep-ph/9912516] [INSPIRE].

[78] J. Guasch, P. Hafliger and M. Spira, MSSM Higgs decays to bottom quark pairs revisited, Phys. Rev. D 68 (2003) 115001 [hep-ph/0305101] [INSPIRE]. 
[79] D. Noth and M. Spira, Supersymmetric Higgs Yukawa couplings to bottom quarks at next-to-next-to-leading order, JHEP 06 (2011) 084 [arXiv:1001.1935] [INSPIRE].

[80] D. Noth and M. Spira, Higgs boson couplings to bottom quarks: two-loop supersymmetry-QCD corrections, Phys. Rev. Lett. 101 (2008) 181801 [arXiv:0808.0087] [INSPIRE].

[81] L. Mihaila and C. Reisser, $O\left(\alpha_{s}^{2}\right)$ corrections to fermionic Higgs decays in the MSSM, JHEP 08 (2010) 021 [arXiv: 1007.0693] [INSPIRE].

[82] E. Bagnaschi et al., Towards precise predictions for Higgs-boson production in the MSSM, JHEP 06 (2014) 167 [arXiv: 1404.0327] [INSPIRE].

[83] M. Carena, S. Heinemeyer, O. Stål, C.E.M. Wagner and G. Weiglein, MSSM Higgs boson searches at the LHC: benchmark scenarios after the discovery of a Higgs-like particle, Eur. Phys. J. C 73 (2013) 2552 [arXiv:1302.7033] [INSPIRE].

[84] P. Bechtle, O. Brein, S. Heinemeyer, G. Weiglein and K.E. Williams, HiggsBounds: confronting arbitrary Higgs sectors with exclusion bounds from LEP and the Tevatron, Comput. Phys. Commun. 181 (2010) 138 [arXiv:0811.4169] [INSPIRE].

[85] P. Bechtle, O. Brein, S. Heinemeyer, G. Weiglein and K.E. Williams, HiggsBounds 2.0.0: confronting neutral and charged Higgs sector predictions with exclusion bounds from LEP and the Tevatron, Comput. Phys. Commun. 182 (2011) 2605 [arXiv:1102.1898] [inSPIRE].

[86] P. Bechtle et al., HiggsBounds-4: improved tests of extended Higgs sectors against exclusion bounds from LEP, the Tevatron and the LHC, Eur. Phys. J. C 74 (2014) 2693 [arXiv: 1311.0055] [INSPIRE].

[87] ATLAS collaboration, Search for new phenomena in final states with large jet multiplicities and missing transverse momentum at $\sqrt{s}=8 \mathrm{TeV}$ proton-proton collisions using the ATLAS experiment, JHEP 10 (2013) 130 [arXiv:1308.1841] [INSPIRE].

[88] CMS collaboration, Search for supersymmetry in hadronic final states with missing transverse energy using the variables $\alpha_{T}$ and b-quark multiplicity in pp collisions at $\sqrt{s}=8 \mathrm{TeV}$, Eur. Phys. J. C 73 (2013) 2568 [arXiv:1303.2985] [InSPIRE].

[89] CMS collaboration, Search for new physics in events with same-sign dileptons and jets in pp collisions at $\sqrt{s}=8 \mathrm{TeV}$, JHEP 01 (2014) 163 [arXiv:1311.6736] [INSPIRE].

[90] S. Heinemeyer, W. Hollik and G. Weiglein, FeynHiggs: a program for the calculation of the masses of the neutral CP even Higgs bosons in the MSSM, Comput. Phys. Commun. 124 (2000) 76 [hep-ph/9812320] [INSPIRE].

[91] M. Frank et al., The Higgs boson masses and mixings of the complex MSSM in the Feynman-diagrammatic approach, JHEP 02 (2007) 047 [hep-ph/0611326] [INSPIRE].

[92] S. Heinemeyer, W. Hollik and G. Weiglein, The masses of the neutral CP-even Higgs bosons in the MSSM: accurate analysis at the two loop level, Eur. Phys. J. C 9 (1999) 343 [hep-ph/9812472] [INSPIRE].

[93] G. Degrassi, P. Slavich and F. Zwirner, On the neutral Higgs boson masses in the MSSM for arbitrary stop mixing, Nucl. Phys. B 611 (2001) 403 [hep-ph/0105096] [InSPIRE].

[94] A. Brignole, G. Degrassi, P. Slavich and F. Zwirner, On the $O\left(\alpha_{t}^{2}\right)$ two loop corrections to the neutral Higgs boson masses in the MSSM, Nucl. Phys. B 631 (2002) 195 [hep-ph/0112177] [INSPIRE].

[95] A. Brignole, G. Degrassi, P. Slavich and F. Zwirner, On the two loop sbottom corrections to the neutral Higgs boson masses in the MSSM, Nucl. Phys. B 643 (2002) 79 [hep-ph/0206101] [INSPIRE]. 
[96] A. Dedes, G. Degrassi and P. Slavich, On the two loop Yukawa corrections to the MSSM Higgs boson masses at large $\tan \beta$, Nucl. Phys. B 672 (2003) 144 [hep-ph/0305127] [INSPIRE].

[97] S. Heinemeyer, W. Hollik, H. Rzehak and G. Weiglein, High-precision predictions for the MSSM Higgs sector at $O\left(\alpha_{b} \alpha_{s}\right)$, Eur. Phys. J. C 39 (2005) 465 [hep-ph/0411114] [INSPIRE].

[98] S. Heinemeyer, W. Hollik, H. Rzehak and G. Weiglein, The Higgs sector of the complex MSSM at two-loop order: QCD contributions, Phys. Lett. B 652 (2007) 300 [arXiv:0705.0746] [INSPIRE].

[99] M. Botje et al., The PDF4LHC working group interim recommendations, arXiv:1101.0538 [INSPIRE].

[100] S. Frixione, New developments in NLOwPS, talk given at the "th Higgs XS WG meeting, December 5-6, CERN, Switzerland (2012).

[101] S. Frixione and B.R. Webber, Matching NLO QCD computations and parton shower simulations, JHEP 06 (2002) 029 [hep-ph/0204244] [INSPIRE].

[102] S. Frixione, Quark mass effects in $g g \rightarrow H$ with $M C @ N L O$, talk given at the ggF meeting on Higgs $p_{T}$, July 23, CERN, Switzerland (2013).

[103] A. Vicini, Quark-mass effects in POWHEG and Hres results, talk given at the ggF meeting on Higgs $p_{T}$, July 23, CERN, Switzerland (2013). 